\title{
VAISMAN SOLVMANIFOLDS AND RELATIONS WITH OTHER GEOMETRIC STRUCTURES*
}

\author{
A. ANDRADA ${ }^{\dagger}$ AND M. ORIGLIA $\ddagger$
}

\begin{abstract}
We characterize unimodular solvable Lie algebras with Vaisman structures in terms of Kähler flat Lie algebras equipped with a suitable derivation. Using this characterization we obtain algebraic restrictions for the existence of Vaisman structures and we establish some relations with other geometric notions, such as Sasakian, coKähler and left-symmetric algebra structures. Applying these results we construct families of Lie algebras and Lie groups admitting a Vaisman structure and we show the existence of lattices in some of these families, obtaining in this way many examples of new solvmanifolds equipped with invariant Vaisman structures.
\end{abstract}

Key words. Locally conformally Kähler structure, Vaisman structure, solvable Lie group, lattice, solvmanifold.

Mathematics Subject Classification. 22E25, 53B35, 53C25, 22E40.

1. Introduction. Let $(M, J, g)$ be a $2 n$-dimensional Hermitian manifold, where $J$ is a complex structure and $g$ is a Hermitian metric, and let $\omega$ denote its fundamental 2 -form, that is, $\omega(X, Y)=g(J X, Y)$ for any $X, Y$ vector fields on $M$. The manifold $(M, J, g)$ is called locally conformally Kähler (LCK) if $g$ can be rescaled locally, in a neighborhood of any point in $M$, so as to be Kähler, i.e., there exists an open covering $\left\{U_{i}\right\}_{i \in I}$ of $M$ and a family $\left\{f_{i}\right\}_{i \in I}$ of $C^{\infty}$ functions, $f_{i}: U_{i} \rightarrow \mathbb{R}$, such that each local metric

$$
g_{i}=\left.\exp \left(-f_{i}\right) g\right|_{U_{i}}
$$

is Kähler. These manifolds are a natural generalization of the class of Kähler manifolds, and they have been much studied by many authors since the work of I. Vaisman in the ' 70 s (see for instance $[19,23,41,52]$ ).

An equivalent characterization of an LCK manifold can be given in terms of the fundamental form $\omega$. Indeed, a Hermitian manifold $(M, J, g)$ is LCK if and only if there exists a closed 1-form $\theta$ globally defined on $M$ such that

$$
d \omega=\theta \wedge \omega .
$$

This closed 1-form $\theta$ is called the Lee form (see [31]). Furthermore, the Lee form $\theta$ is uniquely determined by the following formula:

$$
\theta=-\frac{1}{n-1}(\delta \omega) \circ J
$$

where $\omega$ is the fundamental 2 -form, $\delta$ is the codifferential operator and $2 n$ is the dimension of $M$. A Hermitian manifold $(M, J, g)$ is called globally conformally Kähler (GCK) if there exists a $C^{\infty}$ function, $f: M \rightarrow \mathbb{R}$, such that the metric $\exp (-f) g$ is

${ }^{*}$ Received October 4, 2017; accepted for publication May 7, 2019. This work was partially supported by CONICET, SECyTUNC and ANPCyT (Argentina) and the Research Foundation Flanders (Project G.0F93.17N).

${ }^{\dagger}$ FaMAF-CIEM, Universidad Nacional de Córdoba, Ciudad Universitaria, X5000HUA Córdoba, Argentina (andrada@famaf.unc.edu.ar).

${ }^{\ddagger}$ KU Leuven Kulak, E. Sabbelaan 53, BE-8500 Kortrijk, Belgium; and FaMAF-CIEM, Universidad Nacional de Córdoba, X5000HUA Córdoba, Argentina (origlia@famaf.unc.edu.ar). 
Kähler, or equivalently, the Lee form is exact, $\theta=d f$. Therefore a simply connected LCK manifold is GCK.

It is well known that LCK manifolds belong to the class $\mathcal{W}_{4}$ of the Gray-Hervella classification of almost Hermitian manifolds [24]. Also, an LCK manifold $(M, J, g)$ with $\operatorname{dim} M \geq 4$ is Kähler if and only if $\theta=0$. Indeed, $\theta \wedge \omega=0$ and $\omega$ non degenerate imply $\theta=0$. It is known that if $(M, J, g)$ is a Hermitian manifold with $\operatorname{dim} M \geq 6$ such that (2) holds for some 1 -form $\theta$, then $\theta$ is automatically closed, and therefore $M$ is LCK.

The Hopf manifolds are examples of LCK manifolds, and they are obtained as a quotient of $\mathbb{C}^{n}-\{0\}$ with the Boothby metric by a discrete subgroup of automorphisms. These manifolds are diffeomorphic to $S^{1} \times S^{2 n-1}$ and have first Betti number $b_{1}$ equal to 1 , so that they do not admit any Kähler metric. The LCK structures on these Hopf manifolds have a special property, as shown by Vaisman in [50]. Indeed, the Lee form is parallel with respect to the Levi-Civita connection of the Hermitian metric. The LCK manifolds sharing this property form a distinguished class, which has been much studied since Vaisman's seminal work [23, 29, 42, 43, 50, 51].

Definition 1.1. $(M, J, g)$ is a Vaisman manifold if it is LCK and the Lee form $\theta$ is parallel with respect to the Levi-Civita connection.

A Vaisman manifold satisfies stronger topological properties than general LCK manifolds. For instance, a compact Vaisman non-Kähler manifold $(M, J, g)$ has $b_{1}(M)$ odd $([29,51])$, whereas in [39] an example is given of a compact LCK manifold with even $b_{1}(M)$. This also implies that a compact Vaisman manifold cannot admit Kähler metrics, since the odd Betti numbers of a compact Kähler manifold are even. Moreover, it was proved in [42, Structure Theorem] and [43, Corollary 3.5] that any compact Vaisman manifold admits a Riemannian submersion to a circle such that all fibers are isometric and admit a natural Sasakian structure. It was shown in [53] that any compact complex submanifold of a Vaisman manifold is Vaisman, as well. In [10] the classification of compact complex surfaces admitting a Vaisman structure is given. It is known that a homogeneous LCK manifold is Vaisman when the manifold is compact $([23,28])$ and, more generally, when the manifold is a quotient of a reductive Lie group such that the normalizer of the isotropy group is compact ([2]).

In this article we are interested in invariant Vaisman structures on solvmanifolds, that is, compact quotients $\Gamma \backslash G$ where $G$ is a simply connected solvable Lie group and $\Gamma$ is a lattice in $G$. We begin by studying left invariant Vaisman structures on Lie groups, or equivalently, Vaisman structures on a Lie algebra.

Let $G$ be a Lie group with a left invariant complex structure $J$ and a left invariant metric $g$. If $(G, J, g)$ satisfies the LCK condition $(2)$, then $(J, g)$ is called a left invariant $L C K$ structure on the Lie group $G$. In this case, it follows from (3) that the corresponding Lee form $\theta$ on $G$ is also left invariant.

This fact allows us to define LCK structures on Lie algebras. Recall that a complex structure $J$ on a Lie algebra $\mathfrak{g}$ is an endomorphism $J: \mathfrak{g} \rightarrow \mathfrak{g}$ satisfying $J^{2}=-$ Id and

$$
N_{J}=0, \quad \text { where } \quad N_{J}(x, y)=[J x, J y]-[x, y]-J([J x, y]+[x, J y]),
$$

for any $x, y \in \mathfrak{g}$.

Let $\mathfrak{g}$ be a Lie algebra, $J$ a complex structure and $\langle\cdot, \cdot\rangle$ a Hermitian inner product on $\mathfrak{g}$, with $\omega \in \bigwedge^{2} \mathfrak{g}^{*}$ the fundamental 2-form. We say that $(\mathfrak{g}, J,\langle\cdot, \cdot\rangle)$ is locally conformally Kähler (LCK) if there exists $\theta \in \mathfrak{g}^{*}$, with $d \theta=0$, such that

$$
d \omega=\theta \wedge \omega
$$


Here $d$ denotes the coboundary operator of the Chevalley-Eilenberg complex of $\mathfrak{g}$ corresponding to the trivial representation.

If the Lie group $G$ is simply connected then any left invariant Vaisman structure on $G$ turns out to be globally conformal to a Kähler structure. Therefore we will study compact quotients of such a Lie group by discrete subgroups (if they exist); these quotients will be non simply connected and will inherit a Vaisman structure. Recall that a discrete subgroup $\Gamma$ of a simply connected Lie group $G$ is called a lattice if the quotient $\Gamma \backslash G$ is compact. According to [37], if such a lattice exists then the Lie group must be unimodular. The quotient $\Gamma \backslash G$ is known as a solvmanifold if $G$ is solvable and as a nilmanifold if $G$ is nilpotent, and in these cases we have that $\pi_{1}(\Gamma \backslash G) \cong \Gamma$. Moreover, the diffeomorphism class of solvmanifolds is determined by the isomorphism class of the corresponding lattices, as the following results show:

TheOREM 1.2 ([44, Theorem 3.6]). Let $G_{1}$ and $G_{2}$ be simply connected solvable Lie groups and $\Gamma_{i}, i=1,2$, a lattice in $G_{i}$. If $f: \Gamma_{1} \rightarrow \Gamma_{2}$ is an isomorphism, then there exists a diffeomorphism $F: G_{1} \rightarrow G_{2}$ such that

(i) $\left.F\right|_{\Gamma_{1}}=f$,

(ii) $F(\gamma g)=f(\gamma) F(g)$, for any $\gamma \in \Gamma_{1}$ and $g \in G_{1}$.

COROLlary 1.3 ([38]). Two solvmanifolds with isomorphic fundamental groups are diffeomorphic.

LCK and Vaisman structures on Lie groups and Lie algebras and also on their compact quotients by discrete subgroups have been studied by several authors lately (see $[2,7,8,18,27,28,30,45,46,47,48]$ among others). For instance, it was shown in [45] that if an LCK Lie algebra is nilpotent then it is isomorphic to $\mathfrak{h}_{2 n+1} \times \mathbb{R}$ (where $\mathfrak{h}_{2 n+1}$ is the Heisenberg Lie algebra of dimension $\left.2 n+1\right)$ and the LCK structure is Vaisman (see Example 3.3, cf. [27]). In [2] the authors prove that if a reductive Lie algebra admits an LCK structure then it is isomorphic to either $\mathfrak{u}(2)$ or $\mathfrak{g l}(2, \mathbb{R})$. In [30] it is proved the non-existence of Vaisman metrics on some solvmanifolds with left invariant complex structures. In [8] it is proved that if a nilmanifold $\Gamma \backslash G$ admits a Vaisman structure (not necessarily invariant), then $G$ is isomorphic to the cartesian product of a Heisenberg group $H_{2 n+1}$ with $\mathbb{R}$. In [48] it is shown that if a completely solvable solvmanifold equipped with an invariant complex structure admits a Vaisman metric, then the solvmanifold is again a quotient of $H_{2 n+1} \times \mathbb{R}$. In [34] the authors obtain a Vaisman structure on the total space of certain $S^{1}$-bundles over compact coKähler manifolds, and all the examples they exhibit are diffeomorphic to compact solvmanifolds. Recently, in [3], it was shown that any unimodular Vaisman Lie algebra is isomorphic, up to modifications, to one of the following Lie algebras: $\mathfrak{h}_{2 n+1} \times \mathbb{R}$, $\mathfrak{s u}(2) \times \mathbb{R}$ or $\mathfrak{s l}(2, \mathbb{R}) \times \mathbb{R}$ (see [3] for the relevant definitions).

In this article we obtain a characterization of the unimodular solvable Lie algebras admitting Vaisman structures in terms of Kähler flat Lie algebras equipped with suitable derivations (see Theorems 3.9 and 3.10). More explicitly, we show that any unimodular solvable Vaisman Lie algebra is a double extension of a Kähler flat Lie algebra. In order to do this, we use the fact that Vaisman structures are closely related to Sasakian structures.

This characterization allows us to build new examples of unimodular solvable nonnilpotent Lie algebras equipped with Vaisman structures. When these Lie algebras have integer structural constants we exhibit families of lattices in the associated simply connected solvable Lie groups, and we show that the solvmanifolds obtained in this way are not diffeomorphic to the product of $S^{1}$ with a Heisenberg nilmanifold. 
Moreover, we establish a relation with other geometric structures, namely, with coKähler Lie algebras and left-symmetric algebras. More precisely, we show that any unimodular solvable Vaisman Lie algebra is a central extension of a coKähler flat Lie algebra, and using this we prove the existence of a complete left-symmetric algebra structure on the Vaisman Lie algebra. This gives rise to a complete flat torsion-free connection on any associated solvmanifold.

The article is organized as follows. In $\S 2$ we prove a general result about unimodular LCK Lie algebras and we recall some basic definitions. In $\S 3$ we review some properties about Vaisman Lie algebras and we give the proof of the main theorems (Theorems 3.9 and 3.10). As a consequence of these theorems, we need to study derivations of a Kähler Lie algebra, and we do this in $\S 4$. In $\S 5$, we obtain a strong restriction for the existence of Vaisman structures, namely, if a unimodular solvable Lie algebra admits such a structure then the spectrum of $\operatorname{ad}_{X}$ is contained in $i \mathbb{R}$ for any $X$ in the Lie algebra (see Theorem 5.3). In $\S 6$, using the characterization obtained previously we provide families of new examples of unimodular solvable Vaisman Lie algebras in any even dimension and determine the existence of lattices in many of the corresponding solvable Lie groups. Finally, in $\S 7$ we prove the relation mentioned above with coKähler Lie algebras (Theorem 7.1) and left-symmetric algebras (Corollary 7.4).

Acknowledgments. The authors are grateful to I. Dotti and K. Hasegawa for their useful comments and to the referees for their careful reading of the manuscript and their suggestions.

2. Preliminaries. Let $(\mathfrak{g}, J,\langle\cdot, \cdot\rangle)$ be a Lie algebra with an LCK structure. We have the following orthogonal decomposition for $\mathfrak{g}$

$$
\mathfrak{g}=\mathbb{R} A \oplus \operatorname{ker} \theta
$$

where $\theta$ is the Lee form and $\theta(A)=1$. Since $d \theta=0$, we have that $\mathfrak{g}^{\prime}=[\mathfrak{g}, \mathfrak{g}] \subset \operatorname{ker} \theta$. It is clear that $J A \in \operatorname{ker} \theta$, but when $\mathfrak{g}$ is unimodular we may state a stronger result. Recall that a Lie algebra is unimodular if $\operatorname{tr}\left(\operatorname{ad}_{x}\right)=0$ for all $x$ in the Lie algebra.

Proposition 2.1. If $\mathfrak{g}$ is unimodular and $(J,\langle\cdot, \cdot\rangle)$ is an LCK structure on $\mathfrak{g}$, then $J A \in \mathfrak{g}^{\prime}$.

Proof. Let $\left\{e_{1}, \ldots, e_{2 n}\right\}$ be an orthonormal basis of $\mathfrak{g}$. Recall from [12] the following formula

$$
\delta \eta=-\sum_{i=1}^{2 n} \iota_{e_{i}}\left(\nabla_{e_{i}} \eta\right)
$$

where $\eta$ is a $p$-form and $\delta$ is the codifferential operator. Note that the Koszul formula for the Levi-Civita connection in this setting is given simply by

$$
\left\langle\nabla_{x} y, z\right\rangle=\frac{1}{2}(\langle[x, y], z\rangle-\langle[y, z], x\rangle+\langle[z, x], y\rangle), \quad x, y, z \in \mathfrak{g} .
$$


Using this formula we compute $\delta \omega$, where $\omega$ is the fundamental 2 -form. For $x \in \mathfrak{g}$,

$$
\begin{aligned}
\delta \omega(x) & =-\sum_{i}\left(\nabla_{e_{i}} \omega\right)\left(e_{i}, x\right) \\
& =\sum_{i} \omega\left(\nabla_{e_{i}} e_{i}, x\right)+\omega\left(e_{i}, \nabla_{e_{i}} x\right) \\
& =\sum_{i}-\left\langle\nabla_{e_{i}} e_{i}, J x\right\rangle+\left\langle J e_{i}, \nabla_{e_{i}} x\right\rangle \\
& =\frac{1}{2}\left\{\sum_{i}\left\langle\left[e_{i}, J x\right], e_{i}\right\rangle-\left\langle\left[J x, e_{i}\right], e_{i}\right\rangle+\left\langle\left[e_{i}, x\right], J e_{i}\right\rangle-\left\langle\left[x, J e_{i}\right], e_{i}\right\rangle+\left\langle\left[J e_{i}, e_{i}\right], x\right\rangle\right\} \\
& =\frac{1}{2}\left\{-2 \operatorname{tr}\left(\operatorname{ad}_{J x}\right)+\operatorname{tr}\left(J \circ \operatorname{ad}_{x}\right)-\operatorname{tr}\left(\operatorname{ad}_{x} \circ J\right)+\sum_{i}\left\langle\left[J e_{i}, e_{i}\right], x\right\rangle\right\} \\
& =\frac{1}{2} \sum_{i}\left\langle\left[J e_{i}, e_{i}\right], x\right\rangle .
\end{aligned}
$$

It follows from (3) that $\theta(x)=\frac{1}{2(n-1)} \sum\left\langle J\left[J e_{i}, e_{i}\right], x\right\rangle$. On the other hand, the Lee form can be written in terms of the inner product as $\theta(x)=\frac{\langle A, x\rangle}{|A|^{2}}$. If we compare both expressions we obtain that

$$
A=\frac{|A|^{2}}{2(n-1)} \sum_{i} J\left[J e_{i}, e_{i}\right] .
$$

Therefore $J A \in \mathfrak{g}^{\prime}$.

We will see in forthcoming sections that Vaisman structures on Lie algebras are closely related to certain almost contact metric structures on lower-dimensional Lie algebras. Moreover, when the Vaisman Lie algebra is unimodular and solvable we will show that it is a double extension of a Kähler flat Lie algebra. Let us recall the relevant definitions.

2.1. Almost contact metric Lie algebras. An almost contact metric structure on a Lie algebra $\mathfrak{h}$ is a quadruple $(\langle\cdot, \cdot\rangle, \phi, \xi, \eta)$, where $\langle\cdot, \cdot\rangle$ is an inner product on $\mathfrak{h}, \phi$ is an endomorphism $\phi: \mathfrak{h} \rightarrow \mathfrak{h}$, and $\xi \in \mathfrak{h}, \eta \in \mathfrak{h}^{*}$ satisfy the following conditions:

- $\eta(\xi)=1$,

- $\phi^{2}=-\mathrm{Id}+\eta \otimes \xi$,

- $\langle\phi x, \phi y\rangle=\langle x, y\rangle-\eta(x) \eta(y)$, for all $x, y \in \mathfrak{h}$.

It follows that $|\xi|=1, \phi(\xi)=0, \eta \circ \phi=0$, and $\phi$ is skew-symmetric. The fundamental 2 -form $\Phi$ associated to $(\langle\cdot, \cdot\rangle, \phi, \xi, \eta)$ is defined by $\Phi(x, y)=\langle\phi x, y\rangle$, for $x, y \in \mathfrak{h}$. The almost contact metric structure is called:

- normal if $N_{\phi}=-d \eta \otimes \xi$;

- Sasakian if it is normal and $d \eta=2 \Phi$;

- almost coKähler if $d \eta=d \Phi=0$;

- coKähler if it is almost coKähler and normal (hence $N_{\phi}=0$ ). Equivalently, $\phi$ is parallel (see [14]).

Here $N_{\phi}$ denotes the Nijenhuis tensor associated to $\phi$, which is defined, for $x, y \in \mathfrak{h}$, by

$$
N_{\phi}(x, y)=[\phi x, \phi y]+\phi^{2}[x, y]-\phi([\phi x, y]+[x, \phi y])
$$


CoKähler structures are also known as "cosymplectic", following the terminology introduced by Blair in [13] and used in many articles since then, but their striking analogies with Kähler manifolds have led Li (see [32]) and other authors to use the term "coKähler" for these structures, and this is becoming common practice. In the present article we follow this terminology.

REMARK 2.2. Let $\mathfrak{h}$ be a Lie algebra equipped with a Sasakian structure $(\langle\cdot, \cdot\rangle, \phi, \eta, \xi)$. It follows from $d \eta=2 \Phi$ that $\eta$ is a contact form on $\mathfrak{h}$, and consequently $\xi$ is called the Reeb vector. It is easy to verify that the center of $\mathfrak{h}$ has dimension at most 1 . Moreover, if $\operatorname{dim} \mathfrak{z}(\mathfrak{h})=1$ then the center is generated by the Reeb vector (see [6]).

We recall next a result about Sasakian Lie algebras, which will be necessary to prove our main results.

Proposition $2.3([6])$. Let $(\phi, \eta, \xi,\langle\cdot, \cdot\rangle)$ be a Sasakian structure on a Lie algebra $\mathfrak{h}$ with non trivial center $\mathfrak{z}(\mathfrak{h})$ generated by $\xi$. If $\mathfrak{k}:=\operatorname{ker} \eta$, then the quadruple $\left(\mathfrak{k},[\cdot, \cdot]_{\mathfrak{k}},\left.\phi\right|_{\mathfrak{k}},\left.\langle\cdot, \cdot\rangle\right|_{\mathfrak{k} \times \mathfrak{k}}\right)$ is a Kähler Lie algebra, where $[\cdot, \cdot]_{\mathfrak{k}}$ is the component of the Lie bracket of $\mathfrak{h}$ on $\mathfrak{k}$.

2.2. Double extension of Lie algebras. Let $\mathfrak{h}$ be a real Lie algebra and $\beta \in$ $\bigwedge^{2} \mathfrak{h}^{*}$ a closed 2 -form. If we consider $\mathbb{R}$ as the 1-dimensional abelian Lie algebra, generated by an element $\xi \in \mathbb{R}$, we may define on the vector space $\mathbb{R} \xi \oplus \mathfrak{h}$ the following bracket:

$$
[x, y]_{\beta}=\beta(x, y) \xi+[x, y]_{\mathfrak{h}}, \quad[\xi, x]_{\beta}=0, \quad x, y \in \mathfrak{h} .
$$

It is readily verified that this bracket satisfies the Jacobi identity, and $\mathbb{R} \xi \oplus \mathfrak{h}$ will be called the central extension of $\mathfrak{h}$ by the closed 2 -form $\beta$. It will be denoted $\mathfrak{h}_{\beta}(\xi)$.

Given a derivation $D$ of $\mathfrak{h}_{\beta}(\xi)$, the double extension of $\mathfrak{h}$ by the pair $(D, \beta)$ is defined as the semidirect product $\mathfrak{h}(D, \beta):=\mathbb{R} \ltimes_{D} \mathfrak{h} \beta(\xi)$ (see [4] for more details).

Lemma 2.4. Let $\mathfrak{h}(D, \beta)$ be the double extension of $\mathfrak{h}$ by the pair $(D, \beta)$. Then $\mathfrak{h}(D, \beta)$ is unimodular if and only if $\mathfrak{h}$ is unimodular and $\operatorname{tr} D=0$.

Proof. Let us denote $\mathfrak{g}=\mathfrak{h}(D, \beta)$. Let $A$ be a generator of $\mathbb{R}$, so that $[A, x]=D x$ for all $x \in \mathfrak{h}_{\beta}(\xi)$.

Fix any inner product $\langle\cdot, \cdot\rangle$ on $\mathfrak{g}$ such that $\operatorname{span}\{A, \xi\}$ is orthogonal to $\mathfrak{h},\langle A, \xi\rangle=0$ and $|A|=|\xi|=1$. Given an orthonormal basis $\left\{e_{1}, \ldots, e_{n}\right\}$ of $\mathfrak{h}$, we have that $\{A, \xi\} \cup\left\{e_{1}, \ldots, e_{n}\right\}$ is an orthonormal basis of $\mathfrak{g}$. For any $x \in \mathfrak{h}_{\beta}(\xi)$, we compute

$$
\begin{aligned}
\operatorname{tr}\left(\operatorname{ad}_{x}^{\mathfrak{g}}\right) & =\left\langle[x, A]_{\mathfrak{g}}, A\right\rangle+\left\langle[x, \xi]_{\beta}, \xi\right\rangle+\sum_{i=1}^{n}\left\langle\left[x, e_{i}\right]_{\beta}, e_{i}\right\rangle \\
& =\sum_{i=1}^{n}\left(\left\langle\left[x, e_{i}\right]_{\mathfrak{h}}, e_{i}\right\rangle+\left\langle\beta\left(x, e_{i}\right) \xi, e_{i}\right\rangle\right) \\
& =\operatorname{tr}\left(\operatorname{ad}_{x}^{\mathfrak{h}}\right) .
\end{aligned}
$$

From this and the fact that $\operatorname{tr}\left(\operatorname{ad}_{A}^{\mathfrak{g}}\right)=\operatorname{tr} D$, the result follows. 
3. Vaisman structures on Lie algebras. In this section we show the main results of this article, namely, a characterization of unimodular solvable Lie algebras admitting a Vaisman structure (Theorems 3.9 and 3.10). In order to prove them, we establish first basic properties of Vaisman Lie algebras and later we exploit the close relation between Vaisman and Sasakian structures.

A Vaisman structure on a Lie algebra $\mathfrak{g}$ is an LCK structure $(J,\langle\cdot, \cdot\rangle)$ such that the associated Lee form $\theta$ satisfies $\nabla \theta=0$. The Lie algebra $\mathfrak{g}$ together with the Vaisman structure $(J,\langle\cdot, \cdot\rangle)$ will be called a Vaisman Lie algebra.

If $\mathfrak{g}=\mathbb{R} A \oplus \operatorname{ker} \theta$ with $A \in(\operatorname{ker} \theta)^{\perp}$ such that $\theta(A)=1$, then the Vaisman condition is equivalent to $\nabla A=0$, and since $d \theta=0$, this is in turn equivalent to $A$ being a Killing vector field (considered as a left invariant vector field on the associated Lie group with left invariant metric). Recalling that a left invariant vector field is Killing if and only if the corresponding adjoint operator on the Lie algebra is skew-symmetric, we have:

Proposition $3.1([5])$. If $(J,\langle\cdot, \cdot\rangle)$ is an LCK structure on $\mathfrak{g}$, then it is Vaisman if and only if the endomorphism $\mathrm{ad}_{A}$ is skew-symmetric.

REMARK 3.2. In [47] it was proved that an LCK structure on a unimodular solvable Lie algebra is Vaisman if and only if $\langle[A, J A], J A\rangle=0$, where $A$ is the metric dual of $\theta$. However, the characterization given in Proposition 3.1 will be more useful for our purposes.

EXAMPLE 3.3. Let $\mathfrak{g}=\mathbb{R} \times \mathfrak{h}_{2 n+1}$, where $\mathfrak{h}_{2 n+1}$ is the $(2 n+1)$-dimensional Heisenberg Lie algebra. There is a basis $\left\{x_{1}, \ldots, x_{n}, y_{1}, \ldots, y_{n}, z, w\right\}$ of $\mathfrak{g}$ with Lie brackets given by $\left[x_{i}, y_{i}\right]=z$ for $i=1, \ldots, n$ and $w$ in the center. We define an inner product $\langle\cdot, \cdot\rangle$ on $\mathfrak{g}$ such that the basis above is orthonormal. Let $J$ be the almost complex structure on $\mathfrak{g}$ given by:

$$
J x_{i}=y_{i}, \quad J z=-w \text { for } i=1, \ldots, n .
$$

It is easily seen that $J$ is a complex structure on $\mathfrak{g}$ compatible with $\langle\cdot, \cdot\rangle$. If $\left\{x^{i}, y^{i}, z^{*}, w^{*}\right\}$ denote the 1 -forms dual to $\left\{x_{i}, y_{i}, z, w\right\}$ respectively, then the fundamental 2-form is:

$$
\omega=\sum_{i=1}^{n}\left(x^{i} \wedge y^{i}\right)-z^{*} \wedge w^{*} .
$$

Thus,

$$
d \omega=w^{*} \wedge \omega
$$

and therefore $(\mathfrak{g}, J,\langle\cdot, \cdot\rangle)$ is LCK. It follows from Proposition 3.1 with $A=w$ that this structure is Vaisman. This example appeared in [18] (see also [45, 5]).

It is known that $\mathfrak{g}$ is the Lie algebra of the Lie group $\mathbb{R} \times H_{2 n+1}$, where $H_{2 n+1}$ is the $(2 n+1)$-dimensional Heisenberg group. The Lie group $H_{2 n+1}$ admits a lattice $\Gamma$ and therefore the nilmanifold $N=S^{1} \times \Gamma \backslash H_{2 n+1}$ admits an LCK structure which is Vaisman. It cannot admit any Kähler metric (see [11]), and for $n=1$ the nilmanifold $N$ is a primary Kodaira surface.

The following important properties of Vaisman Lie algebras follow from Proposition 3.1 and the integrability of the complex structure (see also [50]):

Proposition 3.4. Let $(\mathfrak{g}, J,\langle\cdot, \cdot\rangle)$ be a Vaisman Lie algebra, then 
(1) $[A, J A]=0$,

(2) $J \circ \operatorname{ad}_{A}=\operatorname{ad}_{A} \circ J$,

(3) $J \circ \operatorname{ad}_{J A}=\operatorname{ad}_{J A} \circ J$

(4) $\operatorname{ad}_{J A}$ is skew-symmetric.

Without loss of generality, we will assume from now on that $|A|=1$ (rescaling the metric if necessary), hence $\theta(x)=\langle A, x\rangle$ for all $x \in \mathfrak{g}$. Let us denote $W=$ $(\operatorname{span}\{A, J A\})^{\perp}$, so that $\operatorname{ker} \theta=\mathbb{R} J A \oplus^{\perp} W$.

The following proposition, which shows the close relation between Vaisman and Sasakian structures, follows from general results proved by I. Vaisman, but we include a proof at the Lie algebra level for the sake of completeness. We will use the following convention for the action of a complex structure on a 1-form: if $\alpha$ is a 1-form, then $J \alpha:=-\alpha \circ J$.

Proposition 3.5. Set $\xi:=J A, \eta:=\left.J \theta\right|_{\operatorname{ker} \theta}$, and define an endomorphism $\phi \in \operatorname{End}(\operatorname{ker} \theta)$ by $\phi(a \xi+x)=J x$ for $a \in \mathbb{R}$ and $x \in W$. Then the following relations hold:

(1) $\phi^{2}=-\operatorname{Id}+\eta \otimes \xi$,

(2) $\langle\phi x, \phi y\rangle=\langle x, y\rangle-\eta(x) \eta(y)$, for all $x, y \in \operatorname{ker} \theta$,

(3) $N_{\phi}=-d \eta \otimes \xi$,

(4) $d \eta(x, y)=-\langle\phi x, y\rangle$, for all $x, y \in \operatorname{ker} \theta$,

where $N_{\phi}$ is defined as in (5).

Proof. Note that $W=\operatorname{ker} \eta$ and $\eta(\xi)=1$.

(1) and (2) follow from the fact that $(J,\langle\cdot, \cdot\rangle)$ is a Hermitian structure on $\mathfrak{g}$ and $|A|=1$. Since $\mathfrak{g}$ is Vaisman, using Proposition 3.1 and Proposition 3.4, it can be seen that

$$
N_{\phi}(x, y)=N_{J}(x, y)-d \eta(x, y) \xi
$$

for all $x, y \in \operatorname{ker} \theta$. Since $J$ is integrable, we have that $N_{J}=0$ and then we obtain (3).

In order to prove (4) we compute, for all $x, y \in \operatorname{ker} \theta$,

$$
d \eta(x, y)=\theta(J[x, y])=\langle A, J[x, y]\rangle=\omega([x, y], A) .
$$

On the other hand,

$$
\begin{aligned}
\langle J x, y\rangle & =\omega(x, y) \\
& =\theta \wedge \omega(x, y, A) \\
& =d \omega(x, y, A) \\
& =-\omega([x, y], A)-\omega([y, A], x)-\omega([A, x], y) \\
& =-\omega([x, y], A),
\end{aligned}
$$

where we have used Proposition 3.4 in the last step. It is easy to verify that $\langle J x, y\rangle=$ $\langle\phi x, y\rangle$ for any $x, y \in \operatorname{ker} \theta$, thus the proof is complete.

The quadruple $\left(\left.\langle\cdot, \cdot\rangle\right|_{\operatorname{ker} \theta}, \phi, \eta, \xi\right)$ on $\operatorname{ker} \theta$ from Proposition 3.5 does not satisfy exactly the equations of a Sasakian structure given in $\S 2$, but it is easy to show that if we modify it as follows:

$$
\langle\cdot, \cdot\rangle^{\prime}=\frac{1}{4}\langle\cdot, \cdot\rangle, \quad \phi^{\prime}=\phi, \quad \eta^{\prime}=-\frac{1}{2} \eta, \quad \xi^{\prime}=-2 \xi,
$$


then $\left(\langle\cdot, \cdot\rangle^{\prime}, \phi^{\prime}, \eta^{\prime}, \xi^{\prime}\right)$ is a Sasakian structure on $\operatorname{ker} \theta$. However, in this article, for simplicity, we shall call $\left(\left.\langle\cdot, \cdot\rangle\right|_{\operatorname{ker} \theta}, \phi, \eta, \xi\right)$ a Sasakian structure on $\operatorname{ker} \theta$. More generally, when we refer to a Sasakian structure on a Lie algebra we will be assuming that it satisfies the equations on Proposition 3.5. Therefore, we may rewrite Proposition 3.5 as

Corollary 3.6. If $(\mathfrak{g}, J,\langle\cdot, \cdot\rangle)$ is a Vaisman Lie algebra with Lee form $\theta$, then ker $\theta$ has a Sasakian structure.

Conversely, let $\mathfrak{h}$ be a Lie algebra equipped with a Sasakian structure $(\langle\cdot, \cdot\rangle, \phi, \eta, \xi)$. Taking into account Propositions 3.1 and 3.4 we define the Lie algebra $\mathfrak{g}=\mathbb{R} A \ltimes_{D} \mathfrak{h}$ where $D$ is a skew-symmetric derivation of $\mathfrak{h}$ such that $D(\xi)=0$ and $D \phi=\phi D$ on ker $\eta$. We consider on $\mathfrak{g}$ the almost complex structure $J$ given by $\left.J\right|_{\text {ker } \eta}:=\left.\phi\right|_{\operatorname{ker} \eta}, J A=\xi$, and we extend $\langle\cdot, \cdot\rangle$ to an inner product on $\mathfrak{g}$ such that $A$ is orthogonal to $\mathfrak{h}$ and $|A|=1$. Note that $(J,\langle\cdot, \cdot\rangle)$ is an almost hermitian structure on $\mathfrak{g}$. It is easy to prove that $(J,\langle\cdot, \cdot\rangle)$ is in fact a Vaisman structure on $\mathfrak{g}$.

From now on, we assume that $\mathfrak{g}$ is solvable and unimodular (this is a necessary condition for the associated simply connected Lie group to admit lattices, according to [37]). The next step in order to characterize the Lie algebras admitting Vaisman structures is to prove that $J A$ is a central element of $\mathfrak{g}$. Moreover, the dimension of $\mathfrak{z}(\mathfrak{g})$, the center of $\mathfrak{g}$, is at most 2 .

TheOREm 3.7. Let $\mathfrak{g}$ be a unimodular solvable Lie algebra equipped with a Vaisman structure $(J,\langle\cdot, \cdot\rangle)$. Then $J A \in \mathfrak{z}(\mathfrak{g})$. Moreover, $\mathfrak{z}(\mathfrak{g}) \subset \operatorname{span}\{A, J A\}$.

Proof. It follows from Proposition 2.1 that $J A \in \mathfrak{g}^{\prime}$. As $\mathfrak{g}$ is solvable, it follows that $\mathfrak{g}^{\prime}$ is nilpotent and hence $\operatorname{ad}_{J A}: \mathfrak{g} \rightarrow \mathfrak{g}$ is a nilpotent endomorphism. On the other hand, we know that $\operatorname{ad}_{J A}$ is skew-symmetric, according to Proposition 3.4, and therefore $\operatorname{ad}_{J A}=0$, that is, $J A \in \mathfrak{z}(\mathfrak{g})$.

Now we will see that $\mathfrak{z}(\mathfrak{g}) \subset\{A, J A\}$. For $z \in \mathfrak{z}(\mathfrak{g})$, we may assume that $z=$ $a A+z^{\prime}$ with $a \in \mathbb{R}$ and $z^{\prime} \in W$, since $J A \in \mathfrak{z}(\mathfrak{g})$. We have that $0=\operatorname{ad}_{z}=a \operatorname{ad}_{A}+\operatorname{ad}_{z^{\prime}}$, and it follows from Proposition 3.1 that $\operatorname{ad}_{z^{\prime}}$ is a skew-symmetric endomorphism of $\mathfrak{g}$. If $\left[z^{\prime}, J z^{\prime}\right]=c J A+u$ for some $c \in \mathbb{R}, u \in W$, then $c=\left\langle\left[z^{\prime}, J z^{\prime}\right], J A\right\rangle=$ $-\left\langle J z^{\prime},\left[z^{\prime}, J A\right]\right\rangle=0$. On the other hand, taking into account Proposition 3.5, we have that

$$
\begin{aligned}
c & =\left\langle\left[z^{\prime}, J z^{\prime}\right], J A\right\rangle=\eta\left(\left[z^{\prime}, J z^{\prime}\right]\right) \\
& =-d \eta\left(z^{\prime}, J z^{\prime}\right)=\left\langle J z^{\prime}, J z^{\prime}\right\rangle \\
& =\left|z^{\prime}\right|^{2}
\end{aligned}
$$

Therefore $z^{\prime}=0$, and then $z=a A$.

Corollary 3.8. Any unimodular solvable Lie algebra admitting a Sasakian structure has non trivial center.

Proof. Let $\mathfrak{h}$ be a unimodular solvable Lie algebra equipped with a Sasakian structure. Therefore, the semidirect product $\mathfrak{g}=\mathbb{R} A \ltimes_{D} \mathfrak{h}$, where $D$ is a suitable skew-symmetric derivation of $\mathfrak{h}$, admits a Vaisman structure. It is clear that $\mathfrak{g}$ is unimodular and solvable. It follows from Theorem 3.7 that $\mathfrak{h}=\operatorname{ker} \theta$ has non trivial center.

According to Corollary 3.8 the Sasakian Lie algebra ker $\theta$ has non trivial center, which is therefore generated by the Reeb vector $J A$, and $\operatorname{ker} \theta$ can be decomposed 
orthogonally as

$$
\operatorname{ker} \theta=\mathbb{R} J A \oplus W \text {. }
$$

For $x, y \in W$, it is easy to show that

$$
[x, y]=\omega(x, y) J A+[x, y]_{W},
$$

where $[x, y]_{W} \in W$ denotes the component of $[x, y]$ in $W$. It follows from Proposition 2.3 that $\left(W,[\cdot, \cdot]_{W},\left.J\right|_{W},\left.\langle\cdot, \cdot\rangle\right|_{W \times W}\right)$ is a Kähler Lie algebra. We will denote by $\mathfrak{k}$ the Lie algebra $\left(W,[\cdot, \cdot]_{W}\right)$. We point out that $\mathfrak{k}$ is not a Lie subalgebra from either $\operatorname{ker} \theta$ or $\mathfrak{g}$, however, it is clear from (6) that $\operatorname{ker} \theta$ is the central extension $\operatorname{ker} \theta=$ $\mathfrak{k}_{\omega^{\prime}}(J A)$, where $\omega^{\prime}=\left.\omega\right|_{\mathfrak{k} \times \mathfrak{k}}$ is the fundamental 2 -form on $\mathfrak{k}$. Note that $\omega^{\prime}$ is closed since $\left(\mathfrak{k},\left.J\right|_{\mathfrak{k} \times \mathfrak{k}},\left.\langle\cdot, \cdot\rangle\right|_{\mathfrak{k} \times \mathfrak{k}}\right)$ is Kähler. Moreover, if we denote $D:=\left.\operatorname{ad}_{A}\right|_{\operatorname{ker} \theta}$, then $D$ is a skew-symmetric derivation of $\operatorname{ker} \theta$, according to Proposition 3.1. Therefore, $\mathfrak{g}$ can be decomposed orthogonally as

$$
\mathfrak{g}=\mathbb{R} A \ltimes_{D}\left(\mathbb{R} J A \oplus_{\omega^{\prime}} \mathfrak{k}\right),
$$

thus $\mathfrak{g}$ can be regarded as a double extension $\mathfrak{g}=\mathfrak{k}\left(D, \omega^{\prime}\right)$. Note that, according to Proposition 3.4, $D(J A)=0$.

According to Lemma $2.4, \mathfrak{k}$ is unimodular, therefore $\mathfrak{k}$ is a unimodular Lie algebra admitting a Kähler structure $\left(\left.J\right|_{\mathfrak{k}},\left.\langle\cdot, \cdot\rangle\right|_{\mathfrak{k}}\right)$. Due to a classical result from Hano [25], the metric $\left.\langle\cdot, \cdot\rangle\right|_{\mathfrak{k}}$ is flat.

This leads us to use the following terminology: A pair $(\mathfrak{k},\langle\cdot, \cdot\rangle)$ of a Lie algebra $\mathfrak{k}$ equipped with a flat metric $\langle\cdot, \cdot\rangle$ will be called a flat Lie algebra. By abuse of notation, we will say simply sometimes that $\mathfrak{k}$ is a flat Lie algebra. Furthermore, if $(J,\langle\cdot, \cdot\rangle)$ is a Kähler structure on a Lie algebra $\mathfrak{k}$ such that $\langle\cdot, \cdot\rangle$ is flat, then $(\mathfrak{k}, J,\langle\cdot, \cdot\rangle$ ) (or simply $\mathfrak{k}$ ) will be called a Kähler flat Lie algebra.

Resuming our discussion on unimodular solvable Vaisman Lie algebras, we obtain that $\mathfrak{g}$ is a double extension of a Kähler flat Lie algebra.

Let us denote $D^{\prime}:=\left.D\right|_{\mathfrak{k}}$. Then it follows from Proposition 3.4 that $D^{\prime}$ commutes with $\left.J\right|_{\mathfrak{k}}$ and therefore $D^{\prime} \in \mathfrak{u}\left(\mathfrak{k},\left.J\right|_{\mathfrak{k}},\left.\langle\cdot, \cdot\rangle\right|_{\mathfrak{k}}\right)$. Furthermore, $D^{\prime}$ is a derivation of $\mathfrak{k}$. In fact, given $x, y \in \mathfrak{k}$ we have that

$$
\begin{aligned}
D^{\prime}[x, y]_{\mathfrak{k}} & =D([x, y]-\omega(x, y) J A) \\
& =D[x, y] \\
& =[D x, y]+[x, D y] \\
& =\left[D^{\prime} x, y\right]+\left[x, D^{\prime} y\right] \\
& =\omega\left(D^{\prime} x, y\right) J A+\left[D^{\prime} x, y\right]_{\mathfrak{k}}+\omega\left(x, D^{\prime} y\right) J A+\left[x, D^{\prime} y\right]_{\mathfrak{k}} \\
& =\left[D^{\prime} x, y\right]_{\mathfrak{k}}+\left[x, D^{\prime} y\right]_{\mathfrak{k}},
\end{aligned}
$$

since $\omega\left(D^{\prime} x, y\right)=-\omega\left(x, D^{\prime} y\right)$.

Therefore we have associated to any unimodular solvable Vaisman Lie algebra a Kähler flat Lie algebra equipped with a skew-symmetric derivation which commutes with the complex structure. This is summarized in the following theorem.

THEOREM 3.9. Let $\mathfrak{g}$ be a unimodular solvable Lie algebra equipped with a Vaisman structure $(J,\langle\cdot, \cdot\rangle)$, with fundamental 2-form $\omega$ and Lee form $\theta$. Then there exists a Kähler flat Lie algebra $\mathfrak{k}$ such that $\operatorname{ker} \theta=\mathfrak{k}_{\omega^{\prime}}(J A)$ and $\mathfrak{g}=\mathfrak{k}\left(D, \omega^{\prime}\right)$, where 
$D:=\left.\operatorname{ad}_{A}\right|_{\operatorname{ker} \theta}$ and $\omega^{\prime}:=\left.\omega\right|_{\mathfrak{k} \times \mathfrak{k}}$ is the fundamental 2 -form of $\mathfrak{k}$. Moreover, $D^{\prime}:=\left.D\right|_{\mathfrak{k}}$ is a skew-symmetric derivation of $\mathfrak{k}$ that commutes with its complex structure.

Next, we will prove the converse of Theorem 3.9, namely, we show that beginning with a Kähler flat Lie algebra and a suitable derivation we are able to produce a Vaisman structure on a double extension of this Lie algebra.

Theorem 3.10. Let $\left(\mathfrak{k}, J^{\prime},\langle\cdot, \cdot\rangle^{\prime}\right)$ be a Kähler flat Lie algebra with $\omega^{\prime}$ its fundamental 2-form and let $D^{\prime}$ be a skew-symmetric derivation of $\mathfrak{k}$ such that $J^{\prime} D^{\prime}=D^{\prime} J^{\prime}$. Let $D$ be the skew-symmetric derivation of the central extension $\mathfrak{k}_{\omega^{\prime}}(B)$ defined by: $D(B)=0,\left.D\right|_{\mathfrak{k}}=D^{\prime}$. Then the double extension $\mathfrak{g}:=\mathfrak{k}\left(D, \omega^{\prime}\right)=\mathbb{R} A \ltimes_{D} \mathfrak{k}_{\omega^{\prime}}(B)$ admits a Vaisman structure $(J,\langle\cdot, \cdot\rangle)$, where $J A=B,\left.J\right|_{\mathfrak{k}}=J^{\prime}$, and $\langle\cdot, \cdot\rangle$ extends $\langle\cdot, \cdot\rangle^{\prime}$ in the following way: $|A|=|B|=1,\langle A, B\rangle=0,\langle A, \mathfrak{k}\rangle=\langle B, \mathfrak{k}\rangle=0$. The Lie algebra $\mathfrak{g}$ is unimodular and solvable, and the Lee form $\theta$ is the metric dual of $A$.

Proof. Recall that the Lie bracket of $\mathfrak{g}$ is given by: $\left.\operatorname{ad}_{A}\right|_{\mathfrak{k}}=D^{\prime}, B \in \mathfrak{z}(\mathfrak{g})$ and for any $x, y \in \mathfrak{k}$,

$$
[x, y]=\omega^{\prime}(x, y) B+[x, y]_{\mathfrak{k}},
$$

where $\omega^{\prime}(x, y)=\left\langle J^{\prime} x, y\right\rangle^{\prime}$ and $[\cdot, \cdot]_{\mathfrak{k}}$ denotes the Lie bracket on $\mathfrak{k}$. It is easy to see that $(J,\langle\cdot, \cdot\rangle)$ in the statement is an almost Hermitian structure on $\mathfrak{g}$, and we will call $\omega$ its Kähler 2-form. Since $\left(\mathfrak{k},\langle\cdot, \cdot\rangle^{\prime}\right)$ is flat then $\mathfrak{k}$ is unimodular and solvable (see Remark 4.2 below), and it follows from Lemma 2.4 that $\mathfrak{g}$ is unimodular and solvable as well.

Now we show that $J$ is a complex structure on $\mathfrak{g}$. It is enough to show that $N_{J}(x, y)=0$ and $N_{J}(A, y)=0$ for all $x, y \in \mathfrak{k}$. Firstly,

$$
\begin{aligned}
N_{J}(x, y) & =[J x, J y]-[x, y]-J([J x, y]+[x, J y]) \\
& =N_{J^{\prime}}^{\mathfrak{k}}(x, y)+\omega^{\prime}(J x, J y) B-\omega^{\prime}(x, y) B-J\left(\omega^{\prime}(x, J y) B+\omega^{\prime}(J x, y) B\right) \\
& =N_{J^{\prime}}^{\mathfrak{k}}(x, y)+\left(-\left\langle x, J^{\prime} y\right\rangle^{\prime}-\left\langle J^{\prime} x, y\right\rangle^{\prime}\right) B+\left(\left\langle J^{\prime} x, J^{\prime} y\right\rangle^{\prime}-\langle x, y\rangle^{\prime}\right) A \\
& =0,
\end{aligned}
$$

for all $x, y \in \mathfrak{k}$ since $J^{\prime}$ is a complex structure on $\mathfrak{k}$, i.e. $N_{J^{\prime}}^{\mathfrak{k}}=0$. Secondly,

$$
\begin{aligned}
N_{J}(A, y) & =[J A, J y]-[A, y]-J([J A, y]+[A, J y]) \\
& =-D y-J D J y \\
& =-D^{\prime} y-J^{\prime} D^{\prime} J^{\prime} y \\
& =0,
\end{aligned}
$$

for any $y \in \mathfrak{k}$ since $J A=B$ is central and $D^{\prime}$ commutes with $J^{\prime}$. Thus $J$ is integrable on $\mathfrak{g}$.

The next step is to show that $(J,\langle\cdot, \cdot\rangle)$ is LCK, to do this we have to verify that $d \omega=\theta \wedge \omega$ where $\omega$ is the fundamental form on $\mathfrak{g}$ and $\theta$ is the dual 1-form of the vector $A$.

If $x, y, z \in \mathfrak{k}$, then

$$
\begin{aligned}
d \omega(x, y, z) & =-\omega([x, y], z)-\omega([y, z], x)-\omega([z, x], y) \\
& =\left\langle[x, y]_{\mathfrak{k}}+\omega^{\prime}(x, y) B, J z\right\rangle+\left\langle[y, z]_{\mathfrak{k}}+\omega^{\prime}(y, z) B, J x\right\rangle+\left\langle[z, x]_{\mathfrak{k}}+\omega^{\prime}(z, x) B, J y\right\rangle \\
& =\left\langle[x, y]_{\mathfrak{k}}, J^{\prime} z\right\rangle^{\prime}+\left\langle[y, z]_{\mathfrak{k}}, J^{\prime} x\right\rangle^{\prime}+\left\langle[z, x]_{\mathfrak{k}}+, J^{\prime} y\right\rangle^{\prime} \\
& =d^{\mathfrak{k}} \omega^{\prime}(x, y, z) \\
& =0,
\end{aligned}
$$


where $d^{\mathfrak{k}}$ denotes the differential on $\mathfrak{k}$ and we have used that $d^{\mathfrak{k}} \omega^{\prime}=0$. On the other hand, $\theta \wedge \omega(x, y, z)=0$ since $\mathfrak{k} \subset \operatorname{ker} \theta$.

If $y, z \in \mathfrak{k}$, then

$$
\begin{aligned}
d \omega(B, y, z) & =-\omega([B, y], z)-\omega([y, z], B)-\omega([z, B], y) \\
& =\left\langle[y, z]_{\mathfrak{k}}+\omega^{\prime}(y, z) B, J B\right\rangle \\
& =0
\end{aligned}
$$

On the other hand, $\theta \wedge \omega(B, y, z)=0$ since $\mathbb{R} B \oplus \mathfrak{k}=\operatorname{ker} \theta$.

If $y, z \in \mathfrak{k}$, then

$$
\begin{aligned}
d \omega(A, y, z) & =-\omega([A, y], z)-\omega([y, z], A)-\omega([z, A], y) \\
& =\langle D y, J z\rangle+\left\langle\omega^{\prime}(y, z) B+[y, z]_{\mathfrak{k}}, J A\right\rangle+\langle-D z, J y\rangle \\
& =\left\langle D^{\prime} y, J^{\prime} z\right\rangle^{\prime}+\omega^{\prime}(y, z)-\left\langle D^{\prime} z, J^{\prime} y\right\rangle^{\prime} \\
& =\omega^{\prime}(y, z),
\end{aligned}
$$

since $D^{\prime}$ is skew-symmetric and commutes with $J^{\prime}$. On the other hand, $\theta \wedge \omega(A, y, z)=$ $\omega(y, z)=\omega^{\prime}(y, z)$.

If $z \in \mathfrak{k}$, then

$$
\begin{aligned}
d \omega(A, B, z) & =-\omega([A, B], z)-\omega([B, z], A)-\omega([z, A], B) \\
& =\left\langle D^{\prime} z, A\right\rangle^{\prime} \\
& =0
\end{aligned}
$$

since $B$ is central and $D^{\prime} z \in \mathfrak{k}$. On the other hand, $\theta \wedge \omega(A, B, z)=\omega(B, z)=0$.

Thus we have that $(J,\langle\cdot, \cdot\rangle)$ is an LCK structure on $\mathfrak{g}$. Moreover, $(J,\langle\cdot, \cdot\rangle)$ is Vaisman since $\operatorname{ad}_{A}=D$ is a skew-symmetric endomorphism of $\mathfrak{g}$ (Proposition 3.1).

As a by-product of this analysis, we obtain the following stronger version of Corollary 3.8:

Corollary 3.11. Any unimodular solvable Lie algebra admitting a Sasakian structure is a central extension of a Kähler flat Lie algebra.

It follows from Theorems 3.9 and 3.10 that there is a one-to-one correspondence between unimodular solvable Lie algebras equipped with a Vaisman structure and pairs $\left(\mathfrak{k}, D^{\prime}\right)$ where $\mathfrak{k}$ is a Kähler flat Lie algebra and $D^{\prime}$ is a skew-symmetric derivation of $\mathfrak{k}$ which commutes with the complex structure. Moreover, Theorem 3.10 provides a way to construct all unimodular solvable Lie algebras carrying Vaisman metrics. In order to do this, we need a better understanding of the derivations of Kähler flat Lie algebras, and we pursue this in the following section.

4. Derivations of Kähler flat Lie algebras. Let us recall the following result which describes the structure of any Lie algebra equipped with a flat metric. The original version was proved by Milnor in [37], and it was later refined in [9] (compare with [1]).

Proposition $4.1([37],[9])$. Let $(\mathfrak{k},\langle\cdot, \cdot\rangle)$ be a flat Lie algebra. Then $\mathfrak{k}$ decomposes orthogonally as $\mathfrak{k}=\mathfrak{z} \oplus \mathfrak{h} \oplus \mathfrak{k}^{\prime}$ (direct sum of vector spaces) where $\mathfrak{z}$ is the center of $\mathfrak{k}$ and the following properties are satisfied:

(a) $\mathfrak{k}^{\prime}=[\mathfrak{k}, \mathfrak{k}]$ and $\mathfrak{h}$ are abelian. 
(b) ad $: \mathfrak{h} \rightarrow \mathfrak{s o}\left(\mathfrak{k}^{\prime}\right)$ is injective and $\mathfrak{k}^{\prime}$ is even dimensional. In particular, $\operatorname{dim} \mathfrak{h} \leq$ $\frac{\operatorname{dim} \mathfrak{k}^{\prime}}{2}$.

(c) $\operatorname{ad}_{x}=\nabla_{x}$ for any $x \in \mathfrak{z} \oplus \mathfrak{h}$.

(d) $\nabla_{x}=0$ if and only if $x \in \mathfrak{z} \oplus \mathfrak{k}^{\prime}$.

REMARK 4.2. It follows easily from Proposition 4.1 that $\mathfrak{k}$ is a unimodular solvable Lie algebra, whose nilradical is given by $\mathfrak{z} \oplus \mathfrak{k}^{\prime}$.

We will use this proposition in order to better describe Kähler flat Lie algebras. First we give necessary and sufficient conditions for an almost complex structure on a flat Lie algebra to be Kähler (cf. [33]).

Proposition 4.3. Let $(\mathfrak{k},\langle\cdot, \cdot\rangle)$ be a flat Lie algebra and let $J$ be an almost complex structure on $\mathfrak{k}$, compatible with $\langle\cdot, \cdot\rangle$. Then $J$ is Kähler if and only if the following two properties are satisfied:

(i) $\mathfrak{z} \oplus \mathfrak{h}$ and $\mathfrak{k}^{\prime}$ are $J$-invariant.

(ii) $\operatorname{ad}_{H} \circ J=J \circ \operatorname{ad}_{H}$, for any $H \in \mathfrak{h}$.

Proof. Assume first that $J$ is Kähler, that is, $\nabla J=0$, or equivalently $\nabla_{x} J=J \nabla_{x}$ for any $x \in \mathfrak{k}$.

For $x \in \mathfrak{k}^{\prime}$, it follows from Proposition 4.1 that $x=\sum_{i}\left[H_{i}, y_{i}\right]$ for some $H_{i} \in \mathfrak{h}$ and $y_{i} \in \mathfrak{k}^{\prime}$. Then

$$
J x=\sum_{i} J\left[H_{i}, y_{i}\right]=\sum_{i} J \nabla_{H_{i}} y_{i}=\sum_{i} \nabla_{H_{i}} J y_{i}=\sum_{i}\left[H_{i}, J y_{i}\right] \in \mathfrak{k}^{\prime}
$$

where we have used Proposition 4.1(c). Therefore $\mathfrak{k}^{\prime}$ is $J$-invariant. Thus $J$ also preserves the orthogonal complement of $\mathfrak{k}^{\prime}$, that is, $\mathfrak{z} \oplus \mathfrak{h}$ is $J$-invariant, and this proves (i). Finally (ii) follows from the fact that $\nabla_{H} J=J \nabla_{H}$ and $\nabla_{H}=\operatorname{ad}_{H}$ for any $H \in \mathfrak{h}$.

If we assume now that (i) and (ii) hold then it follows easily from Proposition 4.1 that $\nabla_{x} J=J \nabla_{x}$ for any $x \in \mathfrak{k}$, i.e. $J$ is Kähler.

REMARK 4.4. Given any even-dimensional flat Lie algebra, it is easy to define an almost complex structure which satisfies the conditions of Proposition 4.3, thus it becomes a Kähler flat Lie algebra (see $[33,9]$ ).

Our next aim is to study unitary derivations of a Kähler flat Lie algebra, i.e. skew-symmetric derivations which commute with the complex structure. In order to do so, we prove the following lemma.

Lemma 4.5. Let $(\mathfrak{k},\langle\cdot, \cdot\rangle)$ be a flat Lie algebra with decomposition $\mathfrak{k}=\mathfrak{z} \oplus \mathfrak{h} \oplus \mathfrak{k}^{\prime}$ as in Proposition 4.1. If $D$ is a skew-symmetric derivation of $\mathfrak{k}$, then $D(\mathfrak{h})=0$.

Proof. Since $D$ is a derivation of $\mathfrak{k}$, then $D\left(\mathfrak{k}^{\prime}\right) \subset \mathfrak{k}^{\prime}$ and $D(\mathfrak{z}) \subset \mathfrak{z}$. Moreover, since $D$ is skew-symmetric and the sum is orthogonal we have that $D(\mathfrak{h}) \subset \mathfrak{h}$.

It follows from Proposition 4.1(b) that $\operatorname{ad}_{H} \in \mathfrak{s o}\left(\mathfrak{k}^{\prime}\right)$ for any $H \in \mathfrak{h}$. Then, since $\mathfrak{h}$ is abelian, we get that $\mathfrak{F}=\left\{\operatorname{ad}_{H}: \mathfrak{k}^{\prime} \rightarrow \mathfrak{k}^{\prime}: H \in \mathfrak{h}\right\}$ is a commutative family of skew-symmetric endomorphisms of $\mathfrak{k}^{\prime}$. Therefore, $\mathfrak{F}$ is contained in a maximal abelian subalgebra $\mathfrak{a}$ of $\mathfrak{s o}\left(\mathfrak{k}^{\prime}\right)$. The subalgebra $\mathfrak{a}$ is conjugated by an element of $S O\left(\mathfrak{k}^{\prime}\right)$ to the 
following maximal abelian subalgebra of $\mathfrak{s o}\left(\mathfrak{k}^{\prime}\right)$ :

$$
\left\{\left(\begin{array}{ccccc}
0 & -a_{1} & & & \\
a_{1} & 0 & & & \\
& & \ddots & & \\
& & & 0 & -a_{n} \\
& & & a_{n} & 0
\end{array}\right): a_{i} \in \mathbb{R}\right\}
$$

for some orthonormal basis $\left\{e_{1}, f_{1}, \ldots, e_{n}, f_{n}\right\}$ of $\mathfrak{k}^{\prime}$. In this basis the elements of the family $\mathfrak{F}$ can be represented by matrices

$$
\operatorname{ad}_{H}=\left(\begin{array}{ccccc}
0 & -\lambda_{1}(H) & & & \\
\lambda_{1}(H) & 0 & & & \\
& & \ddots & & \\
& & & 0 & -\lambda_{n}(H) \\
& & & \lambda_{n}(H) & 0
\end{array}\right),
$$

for some $\lambda_{i} \in \mathfrak{h}^{*}, i=1, \ldots, n$. We compute

$$
D\left[H, e_{i}\right]=\left[D H, e_{i}\right]+\left[H, D e_{i}\right]=\lambda_{i}(D H) f_{i}+\left[H, D e_{i}\right],
$$

while, on the other hand,

$$
D\left[H, e_{i}\right]=D\left(\lambda_{i}(H) f_{i}\right)=\lambda_{i}(H) D f_{i} .
$$

Comparing the components in the direction of $f_{i}$ in both expressions we obtain that

$$
0=\left\langle\lambda_{i}(H) D f_{i}, f_{i}\right\rangle=\left\langle\lambda_{i}(D H) f_{i}+\left[H, D e_{i}\right], f_{i}\right\rangle=\lambda_{i}(D H),
$$

since $D$ and $\operatorname{ad}_{H}$ are skew-symmetric. Therefore, $\lambda_{i}(D H)=0$ for all $i$, that is, $\operatorname{ad}_{D H}=0$. It follows from Proposition 4.1(b) that $D H=0$.

As a consequence we have the following result, which will be an important tool to produce examples of unimodular solvable Lie algebras with Vaisman structures in $\S 6$.

Theorem 4.6. If $(\mathfrak{k}, J,\langle\cdot, \cdot\rangle)$ is a Kähler flat Lie algebra and $D$ is a unitary derivation of $\mathfrak{k}$, then $D(\mathfrak{h}+J \mathfrak{h})=0$ and there exists an orthonormal basis $\left\{e_{1}, f_{1}, \ldots, e_{n}, f_{n}\right\}$ of $\mathfrak{k}^{\prime}$ such that

$$
\begin{aligned}
& \left.J\right|_{\mathfrak{k}^{\prime}}=\left(\begin{array}{ccccc}
0 & -1 & & & \\
1 & 0 & & & \\
& & \ddots & & \\
& & & 0 & -1 \\
& & & 1 & 0
\end{array}\right),\left.\quad D\right|_{\mathfrak{k}^{\prime}}=\left(\begin{array}{ccccc}
0 & -a_{1} & & & \\
a_{1} & 0 & & & \\
& & \ddots & & \\
& & & 0 & -a_{n} \\
& & & a_{n} & 0
\end{array}\right), \\
& \operatorname{ad}_{H}{\mid \mathfrak{k}^{\prime}}^{\prime}=\left(\begin{array}{ccccc}
0 & -\lambda_{1}(H) & & & \\
\lambda_{1}(H) & 0 & & & \\
& & \ddots & & \\
& & & 0 & -\lambda_{n}(H) \\
& & & \lambda_{n}(H) & 0
\end{array}\right)
\end{aligned}
$$


for some $a_{i} \in \mathbb{R}$ and $\lambda_{i} \in \mathfrak{h}^{*}$ for all $i=1, \ldots, n$. These linear functionals satisfy $\lambda_{i} \neq 0$ for all $i=1, \ldots, n$, and $\bigcap_{i=1}^{n} \operatorname{ker} \lambda_{i}=\{0\}$.

Proof. It follows immediately from Lemma 4.5 and $D J=J D$ that $D(\mathfrak{h}+J \mathfrak{h})=0$.

From Lemma 4.5 and the fact that $D$ is a derivation of $\mathfrak{k}$ we obtain that $D$ commutes with $\operatorname{ad}_{H}$ for all $H \in \mathfrak{h}$. Also, Proposition 4.3 implies that $\left.J\right|_{\mathfrak{k}^{\prime}}$ commutes with $\left.\operatorname{ad}_{H}\right|_{\mathfrak{k}^{\prime}}$ for all $H \in \mathfrak{h}$. Therefore the family $\mathfrak{F}^{\prime}=\left\{\operatorname{ad}_{H}: \mathfrak{k}^{\prime} \rightarrow \mathfrak{k}^{\prime}: H \in \mathfrak{h}\right\} \cup$ $\left\{\left.J\right|_{\mathfrak{k}^{\prime}},\left.D\right|_{\mathfrak{k}^{\prime}}\right\}$ is a commutative family of skew-symmetric endomorphisms of $\mathfrak{k}^{\prime}$. The existence of the basis in the statement follows as in the proof of Lemma 4.5.

We analyze next the linear functionals $\lambda_{i} \in \mathfrak{h}^{*}, i=1, \ldots, n$. If $\lambda_{i}=0$ for some $i$ then $\left[H, e_{i}\right]=0=\left[H, f_{i}\right]$ for all $H \in \mathfrak{h}$, and this implies that $e_{i}, f_{i} \in \mathfrak{z}$, which is a contradiction since $\mathfrak{z} \cap \mathfrak{k}^{\prime}=\{0\}$. Now, if $H \in \bigcap_{i=1}^{n} \operatorname{ker} \lambda_{i}$, we have that $\left.\operatorname{ad}_{H}\right|_{\mathfrak{k}^{\prime}}=0$ and therefore $H=0$ according to Proposition 4.1(b).

5. Further properties of Vaisman Lie algebras. In this section we continue our study of unimodular solvable Vaisman Lie algebras, applying the results obtained in $\S 4$ to the Kähler flat Lie algebra given in Theorem 3.9. In this way we obtain algebraic restrictions for the existence of Vaisman structures.

In Theorem 3.7 we determined the center of a unimodular solvable Lie algebra $\mathfrak{g}$ admitting a Vaisman structure. In what follows we will derive other algebraic properties of these Lie algebras, in particular we will analyze its commutator $\mathfrak{g}^{\prime}$ and its nilradical $\mathfrak{n}$. Recall that, since $\mathfrak{g}$ is solvable, its nilradical is given by $\mathfrak{n}=\{x \in \mathfrak{g}$ : $\operatorname{ad}_{x}: \mathfrak{g} \rightarrow \mathfrak{g}$ is nilpotent $\}$ and $\mathfrak{g}^{\prime} \subseteq \mathfrak{n}$.

Let us set some notation. We denote by $\mathfrak{u}$ the largest $J$-invariant subspace of the center of $\mathfrak{k}$, that is, $\mathfrak{u}=\mathfrak{z} \cap J_{\mathfrak{z}}$, and we define $2 r=\operatorname{dim} \mathfrak{u}+\operatorname{dim} \mathfrak{k}^{\prime}$ and $s=\operatorname{dim} \mathfrak{z}-\operatorname{dim} \mathfrak{u}$. Note that $\mathfrak{z} \oplus \mathfrak{h}$ decomposes orthogonally as $\mathfrak{z} \oplus \mathfrak{h}=\mathfrak{u} \oplus(\mathfrak{h}+J \mathfrak{h})$.

Proposition 5.1. Let $\mathfrak{g}$ be a unimodular solvable Lie algebra admitting a Vaisman structure and consider the decomposition $\mathfrak{g}=\mathbb{R} A \ltimes_{D}\left(\mathbb{R} J A \oplus_{\omega^{\prime}} \mathfrak{k}\right)$ with $\mathfrak{k}=\mathfrak{z} \oplus \mathfrak{h} \oplus \mathfrak{k}^{\prime}$ as in Proposition 4.1. Then the commutator ideal $\mathfrak{g}^{\prime}$ of $\mathfrak{g}$ is given by $\mathfrak{g}^{\prime}=\mathbb{R} J A \oplus \operatorname{Im}\left(\left.D\right|_{\mathfrak{u}}\right) \oplus \mathfrak{k}^{\prime}$, while the nilradical $\mathfrak{n}$ of $\mathfrak{g}$ is given by:

- If $\left.D\right|_{\mathfrak{u}} \neq 0$ or $\left.D\right|_{\mathfrak{k}^{\prime}} \notin \operatorname{ad}(\mathfrak{h}) \subset \mathfrak{s o}\left(\mathfrak{k}^{\prime}\right)$, then $\mathfrak{n}=\mathbb{R} J A \oplus \mathfrak{z} \oplus \mathfrak{k}^{\prime} \simeq \mathbb{R}^{s} \times \mathfrak{h}_{2 r+1}$.

- If $\left.D\right|_{\mathfrak{u}}=0$ and $\left.D\right|_{\mathfrak{k}^{\prime}}=0$, i.e., $A \in \mathfrak{z}(\mathfrak{g})$, then $\mathfrak{n}=\mathbb{R} A \oplus \mathbb{R} J A \oplus \mathfrak{z} \oplus \mathfrak{k}^{\prime} \simeq$ $\mathbb{R}^{s+1} \times \mathfrak{h}_{2 r+1}$.

- If $\left.D\right|_{\mathfrak{u}}=0$ and $0 \neq\left. D\right|_{\mathfrak{k}^{\prime}} \in \operatorname{ad}(\mathfrak{h})$, i.e., $\left.D\right|_{\mathfrak{k}^{\prime}}=-\left.\operatorname{ad}_{H}\right|_{\mathfrak{k}^{\prime}}$ for a unique $0 \neq$ $H \in \mathfrak{h}$, then $\mathfrak{n}=\mathbb{R}(A+H) \oplus \mathbb{R} J A \oplus \mathfrak{z} \oplus \mathfrak{k}^{\prime}$. Furthermore, if $J H \in \mathfrak{h}$, then $\mathfrak{n} \simeq \mathbb{R}^{s+1} \times \mathfrak{h}_{2 r+1} ;$ and if $J H \notin \mathfrak{h}$, then $\mathfrak{n} \simeq \mathbb{R}^{s-1} \times \mathfrak{h}_{2(r+1)+1}$.

Proof. Since $J A \in \mathfrak{g}^{\prime}$ (see Proposition 2.1), it follows from (6) that $\mathfrak{k}^{\prime}=[\mathfrak{k}, \mathfrak{k}]_{\mathfrak{k}} \subset$ $\mathfrak{g}^{\prime}$. Clearly, $D(\mathfrak{h}+J \mathfrak{h})=\left(\left.D\right|_{\mathfrak{k}}\right)(\mathfrak{h}+J \mathfrak{h})$ and, as $\left.D\right|_{\mathfrak{k}}$ is a unitary derivation of $\mathfrak{k}$ (Theorem 3.9), we have $D(\mathfrak{h}+J \mathfrak{h})=0$, due to Theorem 4.6. As a consequence, $\mathfrak{g}^{\prime}=\mathbb{R} J A \oplus \operatorname{Im}\left(\left.D\right|_{\mathfrak{u}}\right) \oplus \mathfrak{k}^{\prime}$

In order to determine the nilradical note first that, for any $z \in \mathfrak{z}, \operatorname{Im}\left(\operatorname{ad}_{z}\right) \subset$ $\mathbb{R} J A \oplus \mathfrak{z}$ and $\operatorname{Im}\left(\operatorname{ad}_{z}^{2}\right) \subset \mathbb{R} J A$. Since $J A \in \mathfrak{z}(\mathfrak{g})$, we obtain that $\operatorname{ad}_{z}$ is a nilpotent operator on $\mathfrak{g}$, which implies that $\mathfrak{z} \subset \mathfrak{n}$. Therefore $\mathbb{R} J A \oplus \mathfrak{z} \oplus \mathfrak{k}^{\prime} \subset \mathfrak{n}$. Moreover, it follows from Proposition 4.1(b) that $\mathfrak{h} \cap \mathfrak{n}=\{0\}$.

Suppose that $\mathbb{R} J A \oplus \mathfrak{z} \oplus \mathfrak{k}^{\prime} \subsetneq \mathfrak{n}$, then there exists $0 \neq A+H \in \mathfrak{n}$ for some $H \in \mathfrak{h}$. Therefore the operator $\operatorname{ad}_{A+H}$ is nilpotent. This operator can be written, for some 
orthonormal basis of $\mathbb{R} J A \oplus \mathfrak{u} \oplus(\mathfrak{h}+J \mathfrak{h}) \oplus \mathfrak{k}^{\prime}$, as

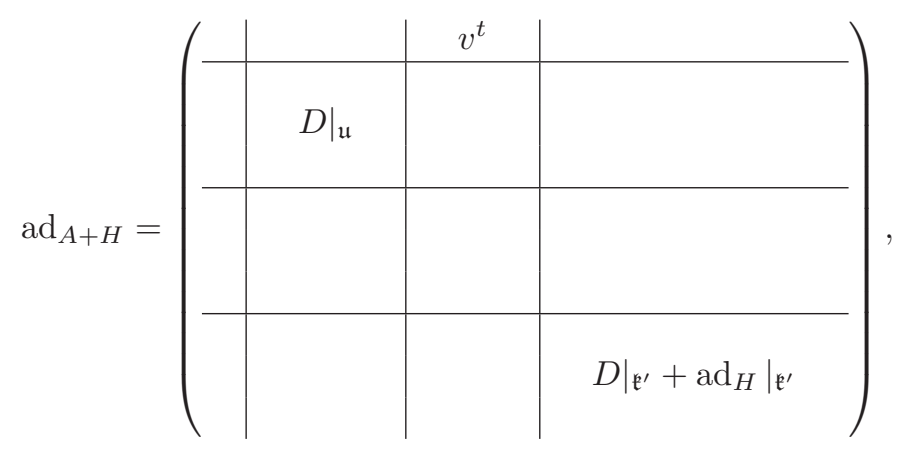

with both $\left.D\right|_{\mathfrak{u}}$ and $\left.D\right|_{\mathfrak{k}^{\prime}}+\left.\operatorname{ad}_{H}\right|_{\mathfrak{k}^{\prime}}$ skew-symmetric. It follows that $\operatorname{ad}_{A+H}$ is nilpotent if and only if

$$
\left.D\right|_{\mathfrak{u}}=0 \quad \text { and }\left.\quad D\right|_{\mathfrak{k}^{\prime}}=-\left.\operatorname{ad}_{H}\right|_{\mathfrak{k}^{\prime}} .
$$

It follows that $H \in \mathfrak{h}$ satisfying (8) is unique, since ad $: \mathfrak{h} \rightarrow \mathfrak{s o}\left(\mathfrak{k}^{\prime}\right)$ is injective (Proposition 4.1(b)). We have two possibilities: if $H=0$, then $A \in \mathfrak{z}(\mathfrak{g})$ and $\mathfrak{n}=$ $\mathbb{R} A \oplus \mathbb{R} J A \oplus \mathfrak{z} \oplus \mathfrak{k}^{\prime}$. On the other hand, if $H \neq 0$, then $\mathfrak{n}=\mathbb{R}(A+H) \oplus \mathbb{R} J A \oplus \mathfrak{z} \oplus \mathfrak{k}^{\prime}$.

Assume now that $\mathfrak{n}=\mathbb{R} J A \oplus \mathfrak{z} \oplus \mathfrak{k}^{\prime}$; according to (8) this happens if and only if $\left.D\right|_{\mathfrak{u}} \neq 0$ or $\left.D\right|_{\mathfrak{k}^{\prime}} \notin \operatorname{ad}(\mathfrak{h})$.

The isomorphism classes of $\mathfrak{n}$ in the different cases above follow easily from the description of the Lie bracket of $\mathfrak{g}$.

REMARK 5.2. Note that the nilradical $\mathfrak{n}$ in Proposition 5.1 is isomorphic to $\mathbb{R}^{p-1} \times \mathfrak{h}_{2 q+1}$, for some $p, q \in \mathbb{N}$ and, as a consequence, it is never abelian. On the other hand, it follows from Proposition 5.1 and Lemma 4.5 that $\mathfrak{n}^{\perp}$ is an abelian subalgebra of $\mathfrak{g}$ if and only if $\mathfrak{h} \cap J \mathfrak{h}=\{0\}$.

We can give next a strong algebraic obstruction to the existence of Vaisman structures on a unimodular solvable Lie algebra.

THEOREM 5.3. If the unimodular solvable Lie algebra $\mathfrak{g}$ admits a Vaisman structure, then the eigenvalues of the operators $\operatorname{ad}_{x}$ with $x \in \mathfrak{g}$ are all imaginary (some of them are 0).

Proof. Recall that $\mathfrak{g}$ is a double extension $\mathfrak{g}=\mathbb{R} A \ltimes_{D}\left(\mathbb{R} J A \oplus_{\omega^{\prime}}\right.$ $\mathfrak{k})$ with $\mathfrak{k}=\mathfrak{z} \oplus \mathfrak{h} \oplus \mathfrak{k}^{\prime}$ and $\mathfrak{z} \oplus \mathfrak{h}=\mathfrak{z} \cap J \mathfrak{z} \oplus(\mathfrak{h}+J \mathfrak{h})$. Let $\left\{A, J A, u_{1}, v_{1}, \ldots, u_{p}, v_{p}, x_{1}, y_{1}, \ldots, x_{q}, y_{q}, e_{1}, f_{1}, \ldots, e_{m}, f_{m},\right\}$ be an orthonormal basis of $\mathfrak{g}$ adapted to this decomposition. Moreover, $J u_{i}=v_{i}, J x_{i}=y_{i}$ and $J e_{i}=f_{i}$.

Given $x=a A+b J A+z+h+y \in \mathfrak{g}$ with $a, b \in \mathbb{R}, z \in \mathfrak{z} \cap J \mathfrak{z}, h \in \mathfrak{h}+J \mathfrak{h}, y \in \mathfrak{k}^{\prime}$, 
we compute the matrix of the operator $\operatorname{ad}_{x}$ in such basis:

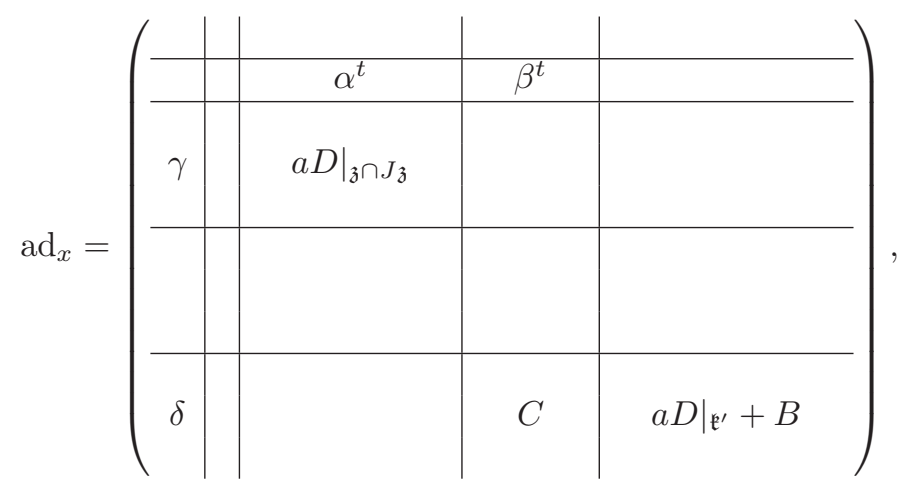

for some $\alpha \in \mathbb{R}^{2 p}, \beta, \gamma \in \mathbb{R}^{2 q}, \delta \in \mathbb{R}^{2 m}, C \in \operatorname{Mat}(m \times q, \mathbb{R})$ and $B \in \mathfrak{u}(m)$. Since $\left.a D\right|_{\mathfrak{z} \cap J \mathfrak{z}}$ and $\left.a D\right|_{\mathfrak{k}^{\prime}}+B$ are skew-symmetric, it is easy to see that the eigenvalues of $\operatorname{ad}_{x}$ for $x \in \mathfrak{g}$ are all imaginary.

REMARK 5.4. A Lie algebra $\mathfrak{g}$ satisfying the condition that the eigenvalues of the operators $\operatorname{ad}_{x}$ are all imaginary for all $x \in \mathfrak{g}$ is called a Lie algebra of type I (see [40]).

A consequence of Theorem 5.3 is the following result, proved recently by H. Sawai in [48]. We recall that a Lie group is called completely solvable if it is solvable and all the eigenvalues of the adjoint operators $\operatorname{ad}_{x}$ are real, for all $x$ in its Lie algebra.

COROLlary 5.5. Let $G$ be a simply connected completely solvable Lie group and $\Gamma \subset G$ a lattice. If the solvmanifold $\Gamma \backslash G$ admits a Vaisman structure $(J, g)$ such that the complex structure $J$ is induced by a left invariant complex structure on $G$, then $G=H_{2 n+1} \times \mathbb{R}$, where $H_{2 n+1}$ denotes the $(2 n+1)$-dimensional Heisenberg Lie group.

Proof. Using the symmetrization process of Belgun ([10]) together with results in [47], one can produce a left invariant Riemannian metric $\tilde{g}$ on $G$ such that $(J, \tilde{g})$ is again a Vaisman structure. This gives rise to a Vaisman structure on $\operatorname{Lie}(G)$ and, taking into account Theorem 5.3 and the fact that $G$ is completely solvable, we have that $G$ is a nilpotent Lie group. It follows from [45] that $G=H_{2 n+1} \times \mathbb{R}$.

In the next section we will show the existence of Vaisman structures on solvmanifolds $\Gamma \backslash G$ where $G$ is not completely solvable, in any even dimension.

6. Examples. Using the results in the previous sections we will construct many examples of unimodular solvable non-nilpotent Lie algebras equipped with Vaisman structures. In particular, we will provide two infinite families of such examples, and also the classification of such Lie algebras in dimensions 4 and 6 . We will also show the existence of families of lattices in these examples, obtaining in this way compact Vaisman solvmanifolds which are not diffeomorphic to the well known nilmanifolds arising from the Heisenberg groups (Example 3.3).

Firstly, we would like to prove a general result concerning lattices in semidirect products, which will be frequently used later in this section.

LEMMA 6.1. Let $H$ be a simply connected Lie group equipped with a Lie group homomorphism $\phi: \mathbb{R} \rightarrow \operatorname{Aut}(H)$, and let $G=\mathbb{R} \ltimes_{\phi} H$ be the corresponding semidirect product. Let $\Gamma$ be a lattice in $H$. If there exists $a \in \mathbb{R}, a \neq 0$, such that $\phi(a)(\Gamma) \subset \Gamma$, then $a \mathbb{Z} \ltimes_{\phi} \Gamma$ is a lattice in $G$. 
Proof. If $a \in \mathbb{R}, a \neq 0$, satisfies $\phi(a)(\Gamma) \subset \Gamma$, then $\phi(a k)(\Gamma) \subset \Gamma$ for all $k \in \mathbb{Z}$ and therefore the semidirect product $\tilde{\Gamma}:=a \mathbb{Z} \ltimes_{\phi} \Gamma$ is well defined. Clearly, $\tilde{\Gamma}$ is a discrete subgroup of $G$. We only have to show that it is co-compact.

Let us recall that two elements $\left(t_{1}, g_{1}\right)$ and $\left(t_{2}, g_{2}\right)$ in $G$ belong to the same rightcoset with respect to $\tilde{\Gamma}$ if and only if there exist $k \in \mathbb{Z}$ and $\gamma \in \Gamma$ such that

$$
\begin{aligned}
\left(t_{2}, g_{2}\right) & =(a k, \gamma)\left(t_{1}, g_{1}\right) \\
& =\left(a k+t_{1}, \gamma \phi(a)^{k} g_{1}\right) .
\end{aligned}
$$

We may assume $a>0$. Let $\left\{a_{n}\right\}$ be a sequence in $\tilde{\Gamma} \backslash G$, with $a_{n}=\left[\left(t_{n}, g_{n}\right)\right]$. It follows easily from (9) that we can choose $t_{n} \in[0, a]$ for all $n \in \mathbb{N}$; moreover, as $\Gamma \backslash H$ is compact, we may assume that $\left[g_{n}\right]$ converges to $[g]$ in $\Gamma \backslash H$, for some $g \in H$.

The canonical projection $\pi: H \rightarrow \Gamma \backslash H$ is a local diffeomorphism, therefore we can choose a representative $g_{n}^{\prime}$ of $\left[g_{n}\right]$ such that $g_{n}^{\prime}$ converges to $g$ in $H$. Taking into account that also $[0, a]$ is compact, we may assume that $a_{n}=\left[\left(t_{n}, g_{n}^{\prime}\right)\right]$ satisfies $t_{n} \rightarrow t$ in $[0, a]$ and $g_{n}^{\prime} \rightarrow g$ in $H$. It is easy to see that $\left[\left(t_{n}, g_{n}^{\prime}\right)\right]$ converges to $[(t, g)]$ in $\tilde{\Gamma} \backslash G$. Since $\left\{a_{n}\right\}$ is arbitrary, we thus obtain that $\tilde{\Gamma} \backslash G$ is compact.

6.1. Example 1. We start with the abelian Lie algebra $\mathfrak{k}=\mathbb{R}^{2 n}$ with its canonical Kähler flat structure $(J,\langle\cdot, \cdot\rangle)$. Let $\left\{e_{1}, f_{1}, \ldots, e_{n}, f_{n}\right\}$ be an orthonormal basis of $\mathfrak{k}$ where $J e_{i}=f_{i}$ and let $\omega=\sum_{i=1}^{n} e^{i} \wedge f^{i}$ be the fundamental form. Then we consider the central extension

$$
\mathfrak{k}_{\omega}(B)=\mathbb{R} B \oplus_{\omega} \mathfrak{k},
$$

which is easily seen to be isomorphic to $\mathfrak{h}_{2 n+1}$, the $(2 n+1)$-dimensional Heisenberg Lie algebra.

Next, we consider the double extension

$$
\mathfrak{g}=\mathfrak{k}(D, \omega)=\mathbb{R} A \ltimes_{D} \mathfrak{h}_{2 n+1},
$$

where the action is given by

$$
D=\left(\begin{array}{cccccc}
0 & & & & & \\
& 0 & -a_{1} & & & \\
& a_{1} & 0 & & & \\
& & & \ddots & & \\
& & & & 0 & -a_{n} \\
& & & & a_{n} & 0
\end{array}\right),
$$

in the basis $\left\{B, e_{1}, f_{1}, \ldots, e_{n}, f_{n}\right\}$ where $\left[e_{i}, f_{i}\right]=B$, for some $a_{i} \in \mathbb{R}$. Since $\left.D\right|_{\mathfrak{k}}$ satisfies the conditions of Theorem $3.10, \mathfrak{g}$ admits a Vaisman structure.

Notice that there is no loss of generality when we assume that $D$ is given as in (10). Indeed, it can be seen that given any unitary derivation $D^{\prime}$ of $\left(\mathbb{R}^{2 n}, J,\langle\cdot, \cdot\rangle\right)$ there exists an orthonormal basis $\left\{e_{1}, f_{1}, \ldots, e_{n}, f_{n}\right\}$ of $\mathbb{R}^{2 n}$ such that $J e_{i}=f_{i}$ and $D^{\prime}=\left.D\right|_{\mathfrak{k}}$ with $D$ is as in (10).

If $a_{i}=0$ for all $i=1, \ldots, n$, then we recover the well known Vaisman structure on $\mathbb{R} \times \mathfrak{h}_{2 n+1}$ (see Example 3.3).

Let us consider from now on the case when not all the $a_{i}$ 's vanish. We may reorder the elements of the basis of $\mathfrak{g}$, if necessary, and we may assume that the constants $a_{i}$ satisfy $a_{1} \leq a_{2} \leq \cdots \leq a_{n}$. We will denote this Lie algebra by $\mathfrak{g}_{\left(a_{1}, \ldots, a_{n}\right)}$. Note 
that $\mathfrak{g}_{\left(a_{1}, \ldots, a_{n}\right)}$ is an almost nilpotent Lie algebra, i.e, it contains a codimension one nilpotent ideal. In particular, the nilradical of $\mathfrak{g}_{\left(a_{1}, \ldots, a_{n}\right)}$ is the nilpotent ideal $\mathfrak{h}_{2 n+1}$. The associated simply connected Lie group will be denoted $G_{\left(a_{1}, \ldots, a_{n}\right)}$ and, when $0<a_{1} \leq a_{2} \leq \cdots \leq a_{n}$, it is known as an oscillator group. Oscillator groups have many geometric properties, for instance, it was proved in [36] that they are the only simply connected, non simple Lie groups which admit an indecomposable bi-invariant Lorentz metric. Other geometric features of oscillator groups in dimension 4 have been studied in [17]. In this example we have shown:

THEOREM 6.2. Any solvmanifold of a group $G_{\left(a_{1}, \ldots, a_{n}\right)}$ admits invariant Vaisman structures. In particular any oscillator solvmanifold admits this kind of structures.

Lattices in oscillator groups have been characterized in [22]. Here we will provide an explicit construction of some families of lattices in $G_{\left(a_{1}, \ldots, a_{n}\right)}$, and later we will determine the first homology group and first Betti number of the associated solvmanifolds.

We begin by recalling the following result, which deals with the isomorphism classes of these Lie algebras.

Lemma 6.3 ([35]). Let $\mathfrak{g}_{\left(a_{1}, \ldots, a_{n}\right)}$ and $\mathfrak{g}_{\left(b_{1}, \ldots, b_{n}\right)}$ be two Lie algebras as defined above. If there exists $c \in \mathbb{R}-\{0\}$ such that $a_{j}=c b_{j}$ for all $j=1, \ldots, n$ then $\mathfrak{g}_{\left(a_{1}, \ldots, a_{n}\right)} \cong \mathfrak{g}_{\left(b_{1}, \ldots, b_{n}\right)}$.

The Lie group $G_{\left(a_{1}, \ldots, a_{n}\right)}$ can be described as follows. We consider the Lie group homomorphism $\varphi: \mathbb{R} \longrightarrow \operatorname{Aut}\left(H_{2 n+1}\right)$ given by

$$
\varphi(t)=e^{t D}=\left(\begin{array}{ccccc}
1 & & & & \\
& \cos \left(a_{1} t\right) & -\sin \left(a_{1} t\right) & & \\
& \sin \left(a_{1} t\right) & \cos \left(a_{1} t\right) & & \\
& & & \ddots & \\
& & & \cos \left(a_{n} t\right) & -\sin \left(a_{n} t\right) \\
& & & \sin \left(a_{n} t\right) & \cos \left(a_{n} t\right)
\end{array}\right)
$$

where $H_{2 n+1}$ is the $(2 n+1)$-dimensional Heisenberg Lie group, i.e. the Euclidean manifold $\mathbb{R}^{2 n+1}$ equipped with the following product:

$$
\begin{aligned}
& \left(z, x_{1}, y_{1}, \ldots, x_{n}, y_{n}\right) \cdot\left(z^{\prime}, x_{1}^{\prime}, y_{1}^{\prime}, \ldots, x_{n}^{\prime}, y_{n}^{\prime}\right) \\
= & \left(z+z^{\prime}+\frac{1}{2} \sum_{j=1}^{n}\left(x_{j} y_{j}^{\prime}-x_{j}^{\prime} y_{j}\right), x_{1}+x_{1}^{\prime}, \ldots, y_{n}+y_{n}^{\prime}\right) .
\end{aligned}
$$

Then $G_{\left(a_{1}, \ldots, a_{n}\right)}$ is the semidirect product $G_{\left(a_{1}, \ldots, a_{n}\right)}=\mathbb{R} \ltimes_{\varphi} H_{2 n+1}$.

We show next the existence of lattices for some choice of the parameters $a_{i}$ (compare [35]):

Proposition 6.4. If $a_{i} \in \mathbb{Q}$ for $i=1, \ldots, n$, then $G_{\left(a_{1}, \ldots, a_{n}\right)}$ admits lattices.

Proof. If $a_{i} \in \mathbb{Q}$, then $a_{i}=\frac{p_{i}}{q_{i}}$ for some $p_{i} \in \mathbb{Z}, q_{i} \in \mathbb{N}$ with $\left(p_{i}, q_{i}\right)=1$. Setting $t_{0}:=2 \pi \prod q_{i}$, we obtain that $\varphi\left(t_{0}\right)$ is an integer matrix. Moreover, the structure constants of $\mathfrak{g}_{\left(a_{1}, \ldots, a_{n}\right)}$ corresponding to the basis $\left\{B, e_{1}, f_{1}, \ldots, e_{n}, f_{n}\right\}$ are all rational. As $G_{\left(a_{1}, \ldots, a_{n}\right)}$ is an almost nilpotent Lie group, it follows from [15] (see also [20]) that $G_{\left(a_{1}, \ldots, a_{n}\right)}$ admits lattices. 
Therefore we will consider $a_{i} \in \mathbb{Q}$ for all $i=1, \ldots, n$; moreover, it follows from Lemma 6.3 that we may assume $a_{i} \in \mathbb{Z}$ for all $i=1, \ldots, n$, with $\operatorname{gcd}\left(a_{1}, \ldots, a_{n}\right)=1$.

Beginning with a lattice in $H_{2 n+1}$ we may extend it to a lattice in $G_{\left(a_{1}, \ldots, a_{n}\right)}$. Consider the following lattices in $H_{2 n+1}$ : for each $k \in \mathbb{N}$ there exists a lattice $\Gamma_{k}$ in $H_{2 n+1}$ given by $\Gamma_{k}=\frac{1}{2 k} \mathbb{Z} \times \mathbb{Z} \times \cdots \times \mathbb{Z}$. It can be shown that $\Gamma_{k} /\left[\Gamma_{k}, \Gamma_{k}\right]$ is isomorphic to $\mathbb{Z}^{2 n} \oplus \mathbb{Z}_{2 k}$. Hence, $\Gamma_{r}$ and $\Gamma_{s}$ are non-isomorphic for $r \neq s$.

Any lattice $\Gamma_{k}$ in $H_{2 n+1}$ is invariant under the subgroups generated by $\varphi(\pi / 2)$, $\varphi(\pi)$ and $\varphi(2 \pi)$. According to Lemma 6.1 we have three families of lattices in $G_{\left(a_{1}, \ldots, a_{n}\right)}$ :

$$
\begin{aligned}
\Lambda_{k, \frac{\pi}{2}} & =\frac{\pi}{2} \mathbb{Z} \ltimes_{\varphi} \Gamma_{k}, \\
\Lambda_{k, \pi} & =\pi \mathbb{Z} \ltimes_{\varphi} \Gamma_{k}, \\
\Lambda_{k, 2 \pi} & =2 \pi \mathbb{Z} \ltimes_{\varphi} \Gamma_{k} .
\end{aligned}
$$

We analyze next some topological properties of the solvmanifolds $\Lambda_{k, j} \backslash G_{\left(a_{1}, \ldots, a_{n}\right)}$ for $j=2 \pi, \pi, \pi / 2$ :

- Note that $\varphi(2 \pi)=\mathrm{Id}$, therefore $\Lambda_{k, 2 \pi}=2 \pi \mathbb{Z} \times \Gamma_{k}$, which is isomorphic to a lattice in $\mathbb{R} \times H_{2 n+1}$. According to Corollary 1.3, we have that the solvmanifold $\Lambda_{k, 2 \pi} \backslash G_{\left(a_{1}, \ldots, a_{n}\right)}$ is isomorphic to the nilmanifold $S^{1} \times \Gamma_{k} \backslash H_{2 n+1}$, for any choice of $\left(a_{1}, \ldots, a_{n}\right)$. It is easy to see that the first homology group of this nilmanifold is $\mathbb{Z}^{2 n+1} \oplus \mathbb{Z}_{2 k}$ and hence its first Betti number is $b_{1}=2 n+1$.

- For the family $\Lambda_{k, \pi}$, note that the isomorphism class of this lattice depends only on the parity of the integers $a_{j}$, therefore according to Corollary 1.3, we may assume that $a_{j} \in\{0,1\}$ for all $j$, not all of them equal to 0 . After reordering, we have that there exists $p \in\{0,1, \ldots, n-1\}$ such that $a_{j}=0$ if $j \leq p$ and $a_{j}=1$ if $j>p$. Then it can be seen that

$$
\begin{aligned}
& {\left[\Lambda_{k, \pi}, \Lambda_{k, \pi}\right]=\left\{\left(0, r, s_{1}, t_{1}, \ldots, s_{n}, t_{n}\right) \in \Lambda_{k, \pi}: r \in \mathbb{Z}\right.} \\
& \left.\quad s_{j}=t_{j}=0(j=1, \ldots, p) ; s_{j}, t_{j} \in 2 \mathbb{Z}(j=p+1, \ldots, n)\right\}
\end{aligned}
$$

thus the first homology group of the solvmanifold $\Lambda_{k, \pi} \backslash G_{\left(a_{1}, \ldots, a_{n}\right)}$ is

$$
\Lambda_{k, \pi} /\left[\Lambda_{k, \pi}, \Lambda_{k, \pi}\right] \cong \mathbb{Z} \oplus \mathbb{Z}_{2 k} \oplus(\mathbb{Z} \oplus \mathbb{Z})^{p} \oplus\left(\mathbb{Z}_{2} \oplus \mathbb{Z}_{2}\right)^{n-p}
$$

The first Betti number is $b_{1}=2 p+1$.

- For the family $\Lambda_{k, \pi / 2}$, note that the isomorphism class of this lattice depends only on the congruence class of the integers $a_{j}$ modulo 4 , therefore according to Corollary 1.3, we may assume that $a_{j} \in\{0,1,2,3\}$ for all $j$, not all of them even. After reordering, we have that there exist $c, d \in\{0,1, \ldots, n-1\}$ with $0 \leq c+d \leq n-1$ such that $a_{1}=\cdots=a_{c}=0, a_{c+1}=\cdots=a_{c+d}=2$ and $a_{j} \in\{1,3\}$ for $j>c+d$. It can be seen that

$$
\begin{array}{r}
{\left[\Lambda_{k, \frac{\pi}{2}}, \Lambda_{k, \frac{\pi}{2}}\right]=\left\{\left(0, r, s_{1}, t_{1}, \ldots, s_{n}, t_{n}\right) \in \Lambda_{k, \frac{\pi}{2}}: r \in \mathbb{Z}, s_{j}=t_{j}=0(j=1, \ldots, c)\right.} \\
\left.s_{j}, t_{j} \in 2 \mathbb{Z}(j=c+1, \ldots, c+d) ; s_{j}+t_{j} \in 2 \mathbb{Z}(j>c+d)\right\}
\end{array}
$$

thus the first homology group of the solvmanifold $\Lambda_{k, \frac{\pi}{2}} \backslash G_{\left(a_{1}, \ldots, a_{n}\right)}$ is

$$
\Lambda_{k, \frac{\pi}{2}} /\left[\Lambda_{k, \frac{\pi}{2}}, \Lambda_{k, \frac{\pi}{2}}\right] \cong \mathbb{Z} \oplus \mathbb{Z}_{2 k} \oplus(\mathbb{Z} \oplus \mathbb{Z})^{c} \oplus\left(\mathbb{Z}_{2} \oplus \mathbb{Z}_{2}\right)^{d} \oplus\left(\mathbb{Z}_{2}\right)^{n-(c+d)} .
$$

The first Betti number is $b_{1}=2 c+1$. 
Clearly, the first homology groups of a solvmanifold in one of the last two families is not isomorphic to the first homology group of the nilmanifolds in the first family. Therefore, the solvmanifolds in the last two families are not diffeomorphic to the nilmanifolds $S^{1} \times \Gamma_{k} \backslash H_{2 n+1}$.

Note that for any $n \in \mathbb{N}$ and $r \in\{0,1, \ldots, n-1\}$, we can find a $(2 n+2)$ dimensional Vaisman solvmanifold $\Lambda_{k, j} \backslash G_{\left(a_{1}, \ldots, a_{n}\right)}$ with first Betti number $b_{1}=2 r+1$.

It is clear that the solvmanifolds we have just constructed admit a Riemannian submersion to the circle $S^{1}$, with fibers isometric to the Sasakian nilmanifold $\Gamma_{k} \backslash H_{2 n+1}$.

REMARK 6.5. In [34] the authors provide examples of compact Vaisman manifolds which are obtained as the total spaces of a principal $S^{1}$-bundle over coKähler manifolds, and they show that they are diffeomorphic to solvmanifolds. The Lie algebras associated to these solvmanifolds are isomorphic to some $\mathfrak{g}_{\left(a_{1}, \ldots, a_{n}\right)}$, but the lattices that they consider are different from ours.

6.2. Example 2. We start with a Kähler flat Lie algebra $(\mathfrak{k}, J,\langle\cdot, \cdot\rangle)$ such that $\operatorname{dim} \mathfrak{h}=1$, where $\mathfrak{k}=\mathfrak{z} \oplus \mathfrak{h} \oplus \mathfrak{k}^{\prime}$ is the orthogonal decomposition of $\mathfrak{k}$ given by Proposition 4.1. Let $\mathfrak{h}=\mathbb{R} H$ with $|H|=1$, and let us set $2 m:=\operatorname{dim} \mathfrak{k}^{\prime}$ and $2 l+1:=\operatorname{dim} \mathfrak{z}$. According to Proposition 4.3, if $Z:=J H$ then $Z \in \mathfrak{z}$ and there exists a $J$-invariant subspace $\mathfrak{u}$ of $\mathfrak{z}$ such that $\mathfrak{z}=\mathbb{R} Z \oplus^{\perp} \mathfrak{u}$. If $\omega$ denotes the fundamental 2 -form of $(J,\langle\cdot, \cdot\rangle)$, then it is easy to verify that the Sasakian central extension of $\mathfrak{k}$ by $\omega$ can be decomposed as:

$$
\mathfrak{k}_{\omega}(B)=\mathbb{R} H \ltimes_{M}\left(\mathbb{R} Z \times \mathfrak{h}_{2(m+l)+1}\right),
$$

where $M$ is the operator given by the following matrix

$$
M=\left(\begin{array}{cccccccc}
0 & 0 & & & & & & \\
1 & 0 & & & & & & \\
& & 0_{2 l \times 2 l} & & & & & \\
& & & 0 & -a_{1} & & & \\
& & a_{1} & 0 & & & \\
& & & & \ddots & & \\
& & & & & 0 & -a_{m} \\
& & & & & & a_{m} & 0
\end{array}\right),
$$

in an orthonormal basis $\left\{Z, B, e_{1}, f_{1}, \ldots, e_{l}, f_{l}, u_{1}, v_{1}, \ldots, u_{m}, v_{m}\right\}$ such that: $\left\{e_{1}, f_{1}, \ldots, e_{l}, f_{l}\right\}$ is a basis of $\mathfrak{u},\left\{u_{1}, v_{1}, \ldots, u_{m}, v_{m}\right\}$ is a basis of $\mathfrak{k}^{\prime}$ and $J e_{i}=f_{i}$, $J u_{i}=v_{i}$. Moreover, $\left[e_{j}, f_{j}\right]=B$ and $\left[u_{j}, v_{j}\right]=B$ for all $j$. It follows from Proposition 4.1 that $a_{j} \neq 0$ for all $j=1, \ldots, m$.

Let $\mathfrak{g}$ be the double extension

$$
\mathfrak{g}=\mathfrak{k}(D, \omega)=\mathbb{R} A \ltimes_{D}\left(\mathbb{R} H \ltimes_{M}\left(\mathbb{R} Z \times \mathfrak{h}_{2(m+l)+1}\right)\right),
$$


where $D$ is the derivation of $\mathfrak{k}_{\omega}(B)$ given by

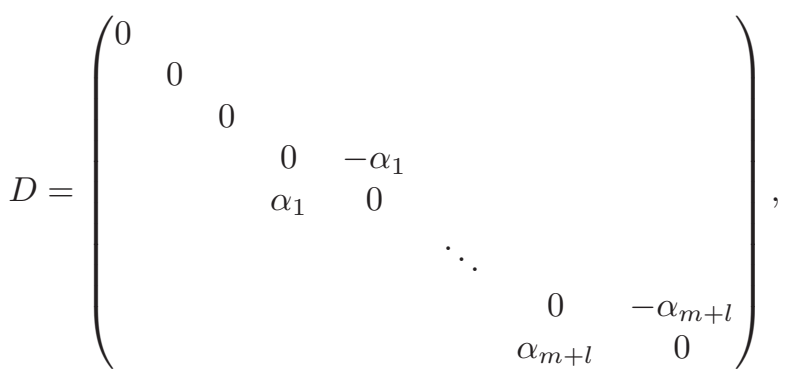

for some $\alpha_{j} \in \mathbb{R}$, in the basis $\left\{H, Z, B, e_{1}, f_{1}, \ldots, e_{l}, f_{l}, u_{1}, v_{1}, \ldots, u_{m}, v_{m}\right\}$. Since $\left.D\right|_{\mathfrak{k}}$ satisfies the conditions of Theorem 3.10 we have that $\mathfrak{g}$ admits a Vaisman structure.

Now we study lattices in the simply connected Lie group $G$ associated to $\mathfrak{g}$ when $a_{i}, \alpha_{i} \in \mathbb{Z}$ for any $i$. Set $n:=m+l$ and consider the Lie group homomorphism $\psi: \mathbb{R} \longrightarrow \operatorname{Aut}\left(\mathbb{R} \times H_{2 n+1}\right)$ given by

$$
\psi(t)=e^{t M}=\left(\begin{array}{ccccccc}
1 & 0 & & & & & \\
t & 1 & & & & & \\
& & \operatorname{Id}_{2 l \times 2 l} & & & \\
& & \cos \left(a_{1} t\right) & -\sin \left(a_{1} t\right) & & \\
& & \sin \left(a_{1} t\right) & \cos \left(a_{1} t\right) & & & \\
& & & \ddots & & \\
& & & & \cos \left(a_{m} t\right) & -\sin \left(a_{m} t\right) \\
& & & & \sin \left(a_{m} t\right) & \cos \left(a_{m} t\right)
\end{array}\right) .
$$

On $\mathbb{R}^{2 n+3}$ consider the algebraic structure given by the semidirect product of $\mathbb{R}$ and $\mathbb{R} \times H_{2 n+1}$ via $\psi$ and we obtain the simply connected Lie group

$$
S=\mathbb{R} \ltimes_{\psi}\left(\mathbb{R} \times H_{2 n+1}\right) .
$$

If $\Gamma_{k}$ is the lattice in $H_{2 n+1}$ considered in the examples in $\S 6.1$, then the group $L_{k}=a \mathbb{Z} \times \Gamma_{k}$ is a lattice in $\mathbb{R} \times H_{2 n+1}$ for any $a \in \mathbb{R}, a \neq 0$. In particular, according to Lemma 6.1 , for each $j=2 \pi, \pi, \frac{\pi}{2}$ we obtain a lattice $\Gamma_{k, j}$ in $S$ defined by

$$
\Gamma_{k, j}=j \mathbb{Z} \ltimes\left(j^{-1} \mathbb{Z} \times \Gamma_{k}\right) .
$$

Now we consider the Lie group homomorphism $\varphi: \mathbb{R} \longrightarrow \operatorname{Aut}(S)$ given by

$$
\varphi(t)=e^{t D}=\left(\begin{array}{ccccccc}
1 & & & & & & \\
& 1 & & & & & \\
& & 1 & & & & \\
& & \cos \left(\alpha_{1} t\right) & -\sin \left(\alpha_{1} t\right) & & \\
& & \sin \left(\alpha_{1} t\right) & \cos \left(\alpha_{1} t\right) & & \\
& & & & \ddots & & \\
& & & & & \cos \left(\alpha_{n} t\right) & -\sin \left(\alpha_{n} t\right) \\
& & & & & \sin \left(\alpha_{n} t\right) & \cos \left(\alpha_{n} t\right)
\end{array}\right) .
$$

On $\mathbb{R}^{2 n+4}$ consider the algebraic structure given by the semidirect product of $\mathbb{R}$ and $S$ via $\varphi$ and we obtain the simply connected Lie group

$$
G=\mathbb{R} \ltimes_{\varphi} S .
$$


Since $\Gamma_{k, j}$ is invariant under the subgroups generated by $\varphi(2 \pi), \varphi(\pi), \varphi\left(\frac{\pi}{2}\right)$, then for each $\Gamma_{k, j}$ we have three new lattices in $G$, given by

$$
\Lambda_{k, j, i}=i \mathbb{Z} \ltimes \Gamma_{k, j},
$$

for $i=2 \pi, \pi, \frac{\pi}{2}$. Therefore we get new examples of solvmanifolds

$$
M_{k, j, i}=\Lambda_{k, j, i} \backslash \mathbb{R} \ltimes_{\varphi}\left(\mathbb{R} \ltimes_{\psi}\left(\mathbb{R} \times H_{2 n+1}\right)\right)
$$

equipped with a Vaisman structure arising from a Vaisman structure on the Lie algebra.

The solvmanifolds we have just constructed admit a Riemannian submersion to the circle $S^{1}$, with fibers isometric to a Sasakian solvmanifold $\Gamma_{k, j} \backslash\left(\mathbb{R} \ltimes_{\psi}\left(\mathbb{R} \times H_{2 n+1}\right)\right)$.

6.3. Classification of Vaisman Lie algebras in low dimensions. In this section we determine all unimodular solvable Lie algebras of dimension 4 and 6 that admit a Vaisman structure, up to Lie algebra isomorphism.

(i) According to Theorem 3.9, any 4-dimensional unimodular solvable Lie algebra with a Vaisman structure is a double extension $\mathfrak{g}=\mathfrak{k}(D, \omega)$, where $\mathfrak{k}=\mathbb{R}^{2}$. It is easy to see that $\mathfrak{g}$ has an orthonormal basis $\{A, B, e, f\}$ such that

$$
[A, e]=c f, \quad[A, f]=-c f, \quad[e, f]=B, \quad J A=B, J e=f, \quad c \in \mathbb{R} .
$$

These Lie algebras belong to the family of examples constructed in $\S 6.1$. If $c=0$, then $\mathfrak{g}$ is isomorphic to the nilpotent Lie algebra $\mathbb{R} \times \mathfrak{h}_{3}$, equipped with its canonical Vaisman structure (see Example 3.3), and any nilmanifold obtained as a quotient of $\mathbb{R} \times H_{3}$ is a primary Kodaira surface. If $c \neq 0$, then we may assume $c=1$ (via a Lie algebra isomorphism), and $\mathfrak{g}$ is a solvable non-nilpotent Lie algebra $\mathbb{R} \ltimes \mathfrak{h}_{3}$, and any solvmanifold obtained as a quotient of $\mathbb{R} \ltimes H_{3}$ is either a primary or secondary Kodaira surface, depending on the lattice considered (see [26] and §6.1).

(ii) According to Theorem 3.9, any 6-dimensional unimodular solvable Lie algebra with a Vaisman structure is a double extension $\mathfrak{g}=\mathfrak{k}(D, \omega)$, where $\mathfrak{k}$ is a 4-dimensional Kähler flat Lie algebra which decomposes orthogonally as $\mathfrak{k}=\mathfrak{z} \oplus \mathfrak{h} \oplus \mathfrak{k}^{\prime}$, according to Proposition 4.1. In particular, the dimension of $\mathfrak{k}^{\prime}$ must be 0 or 2 and, as a consequence, $\operatorname{dim} \mathfrak{h}$ is equal to 0 or 1 . Therefore there are only two cases for $\mathfrak{k}$, namely, $\mathfrak{k}_{1}=\mathfrak{z}=\mathbb{R}^{4}$ or $\mathfrak{k}_{2}=\mathbb{R} Z \oplus \mathbb{R} H \oplus \mathbb{R}^{2}$ for some $Z \in \mathfrak{z}, H \in \mathfrak{h}$ with $J H=Z$. In the latter case, the action of $H$ on $\mathfrak{k}^{\prime}$ is given by a matrix $\left(\begin{array}{cc}0 & -a \\ a & 0\end{array}\right)$, with $a \neq 0$, and it is easy to see that these Lie algebras are all isomorphic to the one with $a=1$. The Sasakian central extension of $\mathfrak{k}_{1}$ is isomorphic to $\mathfrak{h}_{5}$, while the Sasakian central extension of $\mathfrak{k}_{2}$ is the Lie algebra $\mathfrak{s}_{5}$ generated by $\{B, Z, H, e, f\}$ with Lie brackets

$$
[H, e]=f,[H, f]=-e,[H, Z]=[e, f]=B,
$$

where $\{e, f\}$ is an orthonormal basis of $\mathbb{R}^{2}$ with $J e=f$. It is a consequence of Corollary 3.11 that $\mathfrak{h}_{5}$ and $\mathfrak{s}_{5}$ are the only unimodular solvable Lie algebras of dimension 5 which admit Sasakian structures (this follows also from [6]).

The corresponding unimodular solvable Vaisman Lie algebras, obtained as double extensions of $\mathfrak{k}_{1}$ and $\mathfrak{k}_{2}$, are given in the next result. Note that the double extensions $\mathfrak{k}_{1}(D, \omega)$ belong to the family in $\S 6.1$, while the double extensions $\mathfrak{k}_{2}(D, \omega)$ belong to the family in $\S 6.2$.

Proposition 6.6. Let $\mathfrak{g}$ be a 6-dimensional unimodular solvable Lie algebra. If $\mathfrak{g}$ admits a Vaisman structure, then $\mathfrak{g}$ is isomorphic to one of the following Lie algebras: 
(i) $\mathbb{R} \times \mathfrak{h}_{5}$ or $\mathbb{R} \ltimes_{D_{r}} \mathfrak{h}_{5}$ for $r \in[-1,1]$, where $\mathfrak{h}_{5}=\operatorname{span}\left\{B, e_{1}, e_{2}, e_{3}, e_{4}\right\}$, with Lie brackets given by: $\left[e_{1}, e_{2}\right]=\left[e_{3}, e_{4}\right]=B$ and

$$
D_{r}=\left(\begin{array}{ccccc}
0 & & & & \\
& 0 & -r & & \\
& r & 0 & & \\
& & & 0 & -1 \\
& & & 1 & 0
\end{array}\right)
$$

in this basis.

(ii) $\mathbb{R} \times \mathfrak{s}_{5}$ or $\mathbb{R} \ltimes_{D_{0}} \mathfrak{s}_{5}$ where $\mathfrak{s}_{5}=\operatorname{span}\left\{B, e_{1}, e_{2}, e_{3}, e_{4}\right\}$, with Lie brackets given by: $\left[e_{1}, e_{3}\right]=e_{4},\left[e_{1}, e_{4}\right]=-e_{3},\left[e_{1}, e_{2}\right]=\left[e_{3}, e_{4}\right]=B$ and $D_{0}$ is as in $(i)$ with $r=0$.

Moreover, all these Lie algebras are pairwise non-isomorphic and the corresponding simply connected Lie groups admit lattices (with $r \in \mathbb{Q}$ for $\mathbb{R} \ltimes_{D_{r}} \mathfrak{h}_{5}$ ).

Proof. It is easy to see, taking into account Lemma 6.3 , that $\mathfrak{k}_{1}(D, \omega)$ is isomorphic to either $\mathbb{R} \times \mathfrak{h}_{5}$ or $\mathbb{R} \ltimes_{D_{r}} \mathfrak{h}_{5}$ with $r \in[-1,1]$. It can be verified that these Lie algebras are pairwise non-isomorphic. On the other hand, for $\mathfrak{k}_{2}(D, \omega)$, using Theorem 4.6 it can be easily seen that this Lie algebra is isomorphic to either $\mathbb{R} \times \mathfrak{s}_{5}$ or $\mathbb{R} \ltimes_{D_{0}} \mathfrak{s}_{5}$. Using Proposition 5.1 and after some computations, it can be seen that $\mathbb{R} \times \mathfrak{s}_{5}$ or $\mathbb{R} \ltimes_{D_{0}} \mathfrak{s}_{5}$ are not isomorphic to neither $\mathbb{R} \times \mathfrak{h}_{5}$ nor $\mathbb{R} \ltimes_{D_{r}} \mathfrak{h}_{5}$.

Note that $\mathbb{R} \ltimes_{D_{r}} \mathfrak{h}_{5}$ corresponds to the Lie algebra $\mathfrak{g}_{(r, 1)}$ with $r \in[-1,1]$ studied in §6.1. Therefore the existence of lattices for the group $G_{(r, 1)}$ with $r \in \mathbb{Q}$ was established in Proposition 6.4. In the case of $\mathbb{R} \times \mathfrak{s}_{5}$ and $\mathbb{R} \ltimes_{D_{0}} \mathfrak{s}_{5}$ the existence of lattices in the associated simply connected Lie group follows from $\S 6.2$ (see also [6]).

It follows from Lemma 6.3 that the Lie algebra $\mathfrak{g}_{(r, 1)}=\mathbb{R} \ltimes_{D_{r}} \mathfrak{h}_{5}, r \in \mathbb{Q}$, from Proposition 6.6 is isomorphic to $\mathfrak{g}_{(a, b)}$ for some $a, b \in \mathbb{Z},(a, b)=1$. Next we determine the first homology group and the first Betti number of the 6 -dimensional solvmanifolds $\Lambda_{k, j} \backslash G_{(a, b)}$ for $j=2 \pi, \pi, \pi / 2$, following $\S 6.1$.

- For the familiy $\Lambda_{k, 2 \pi}=2 \pi \mathbb{Z} \times \Gamma_{k}$, the solvmanifold $\Lambda_{k, 2 \pi} \backslash G_{(a, b)}$ is diffeomorphic to a nilmanifold $S^{1} \times \Gamma_{k} \backslash H_{5}$, for all $(a, b)$. The first homology group of this nilmanifold is $\mathbb{Z}^{5} \oplus \mathbb{Z}_{2 k}$ and hence its first Betti number is $b_{1}=5$.

- For the family $\Lambda_{k, \pi}$, the isomorphism class of this lattice depends only on the parity of the integers $a, b$. Then it is straightforward to verify that the first homology group of the solvmanifold $M:=\Lambda_{k, \pi} \backslash G_{(a, b)}$ and its first Betti number are given by:

\begin{tabular}{|c|c|c|}
\hline$a, b$ & $H_{1}(M, \mathbb{Z})$ & $b_{1}(M)$ \\
\hline$a b \equiv 1(\bmod 2)$ & $\mathbb{Z} \oplus \mathbb{Z}_{2 k} \oplus \mathbb{Z}_{2}^{4}$ & 1 \\
\hline$a b \equiv 0(\bmod 2)$ & $\mathbb{Z}^{3} \oplus \mathbb{Z}_{2 k} \oplus \mathbb{Z}_{2}^{2}$ & 3 \\
\hline
\end{tabular}

- For the family $\Lambda_{k, \pi / 2}$, the isomorphism class of this lattice depends only on the congruence class of the integers $a, b$ modulo 4 , and it can be seen that the first homology group of the solvmanifold $M:=\Lambda_{k, \frac{\pi}{2}} \backslash G_{(a, b)}$ and its first Betti number are given by: 


\begin{tabular}{|c|c|c|}
\hline$a, b$ & $H_{1}(M, \mathbb{Z})$ & $b_{1}(M)$ \\
\hline$a b \equiv \pm 1(\bmod 4)$ & $\mathbb{Z} \oplus \mathbb{Z}_{2 k} \oplus \mathbb{Z}_{2}^{2}$ & 1 \\
\hline$a b \equiv 2(\bmod 4)$ & $\mathbb{Z} \oplus \mathbb{Z}_{2 k} \oplus \mathbb{Z}_{2}^{3}$ & 1 \\
\hline$a b \equiv 0(\bmod 4)$ & $\mathbb{Z}^{3} \oplus \mathbb{Z}_{2 k} \oplus \mathbb{Z}_{2}$ & 3 \\
\hline
\end{tabular}

\section{Relation with other geometric structures.}

7.1. CoKähler Lie algebras. In previous sections we have seen that any unimodular solvable Lie algebra admitting a Vaisman structure is the semidirect product of $\mathbb{R}$ with a Sasakian Lie algebra and this Sasakian Lie algebra, in turn, is a central extension of a Kähler flat Lie algebra. In what follows we will establish a relation with another type of almost contact metric Lie algebras, namely, coKähler ones. In fact, we will show that a unimodular solvable Lie algebra admitting a Vaisman structure is a central extension of a coKähler Lie algebra which is, in turn, a semidirect product of $\mathbb{R}$ with a Kähler flat Lie algebra.

Let $\mathfrak{g}$ be a unimodular solvable Lie algebra that admits a Vaisman structure $(J,\langle\cdot, \cdot\rangle)$, with $\omega$ its fundamental 2-form, $\theta$ the corresponding Lee form and $A \in \mathfrak{g}$ its metric dual, as before. We know from Theorem 3.9 that $\mathfrak{g}$ is a double extension $\mathfrak{g}=\mathfrak{k}\left(D, \omega^{\prime}\right)$ of the Kähler flat Lie algebra $\mathfrak{k}$ by certain derivation $D$ of $\mathfrak{k}_{\omega^{\prime}}(\xi)$ such that $D^{\prime}:=\left.D\right|_{\mathfrak{k}}$ is a unitary derivation of $\mathfrak{k}$.

TheOREM 7.1. With notation as above, the Lie algebra $\mathfrak{d}=\mathbb{R} A \ltimes_{D^{\prime}} \mathfrak{k}$ admits a coKähler structure $\left(\left.\langle\cdot, \cdot\rangle\right|_{\mathfrak{d} \times \mathfrak{d}}, \phi, \xi, \eta\right)$, where $\phi \in \operatorname{End}(\mathfrak{d})$ is defined by $\phi(a A+x)=$ Jx for $a \in \mathbb{R}, x \in \mathfrak{k}$, and $\eta:=\left.\theta\right|_{\mathfrak{d}}, \xi:=A$. Moreover, if $\Phi$ denotes the (closed) fundamental 2-form on $\mathfrak{d}$ associated to this coKähler structure, then $\mathfrak{g}$ is isomorphic to the central extension $\mathfrak{d}_{\Phi}(J A)$.

Proof. It is readily verified that $\left(\left.\langle\cdot, \cdot\rangle\right|_{\mathfrak{o} \times \mathfrak{o}}, \phi, \xi, \eta\right)$ is an almost contact metric structure on $\mathfrak{d}$. Let us prove now that it is almost coKähler, i.e., $d \eta=0$ and $d \Phi=0$, where $\Phi$ is the fundamental 2 -form defined by $\Phi(x, y)=\langle\phi x, y\rangle, x, y \in \mathfrak{d}$.

Since $[\mathfrak{d}, \mathfrak{d}] \subset \mathfrak{k}=\operatorname{ker} \eta$, we have that $d \eta=0$. Now, for $a, b, c \in \mathbb{R}$ and $x, y, z \in \mathfrak{k}$, we compute easily

$$
\begin{aligned}
& d \Phi(a A+x, b A+y, c A+z) \\
& =d^{\mathfrak{k}} \omega^{\prime}(x, y, z)+a\left(\left\langle D^{\prime} y, J z\right\rangle-\left\langle D^{\prime} z, J y\right\rangle\right) \\
& \quad+b\left(\left\langle D^{\prime} z, J x\right\rangle-\left\langle D^{\prime} x, J z\right\rangle\right)+c\left(\left\langle D^{\prime} x, J y\right\rangle-\left\langle D^{\prime} y, J x\right\rangle\right),
\end{aligned}
$$

where $\omega^{\prime}=\left.\omega\right|_{\mathfrak{k} \times \mathfrak{k}}$. Since $\omega^{\prime}$ is the Kähler form on $\mathfrak{k}$, we have that $d^{\mathfrak{k}} \omega^{\prime}=0$. Since both $D$ and $J$ are skew-symmetric and $\left.D^{\prime} J\right|_{\mathfrak{k}}=\left.J\right|_{\mathfrak{k}} D^{\prime}$, we obtain that the last terms in (11) vanish, and therefore $d \Phi=0$.

To verify the normality of this structure, since $d \eta=0$ we only have to check that $N_{\phi}=0$. For $a, b \in \mathbb{R}$ and $x, y \in \mathfrak{k}$, we compute

$$
N_{\phi}(a A+x, b A+y)=N_{\left.J\right|_{\mathfrak{k}}}^{\mathfrak{k}}(x, y)-a\left(D^{\prime} y+J D^{\prime} J y\right)+b\left(D^{\prime} x+J D^{\prime} J x\right) .
$$

Since $N_{\left.J\right|_{\mathfrak{k}}}^{\mathfrak{k}}=0$ and $\left.D^{\prime} J\right|_{\mathfrak{k}}=\left.J\right|_{\mathfrak{k}} D^{\prime}$, it follows that $N_{\phi}=0$.

To prove the last statement we compute the Lie bracket $[\cdot, \cdot]^{\prime}$ on the central 
extension $\mathfrak{d}_{\Phi}(J A)$. We have that $J A$ is central and for $a, b \in \mathbb{R}, x, y \in \mathfrak{k}$ we compute

$$
\begin{aligned}
{[a A+x, b A+y]^{\prime} } & =\Phi(a A+x, b A+y) J A+[a A+x, b A+y]_{\mathfrak{o}} \\
& =\langle\phi(a A+x), b A+y\rangle J A+a D^{\prime} y-b D^{\prime} x+[x, y]_{\mathfrak{k}} \\
& =\langle J x, y\rangle J A+a D y-b D x+[x, y]_{\mathfrak{k}} \\
& =\omega(x, y) J A+a D y-b D x+[x, y]_{\mathfrak{k}},
\end{aligned}
$$

which coincides with the Lie bracket on $\mathfrak{g}$, according to Theorem 3.7 and (6). This completes the proof.

Remark 7.2. The first part of Theorem 7.1 follows also from [21, Theorem 6.1]. Moreover, according to [21, Proposition 6.4], $(\mathfrak{d},\langle\cdot, \cdot\rangle)$ is a flat Lie algebra, since $\mathfrak{d}$ is unimodular. If, as above, $\mathfrak{k}=\mathfrak{z} \oplus \mathfrak{h} \oplus \mathfrak{k}^{\prime}$ is the decomposition of $\mathfrak{k}$ given by Proposition 4.1 , then the corresponding decomposition of $\mathfrak{d}$ is $\mathfrak{d}=\widetilde{\mathfrak{z}} \oplus \widetilde{\mathfrak{h}} \oplus \mathfrak{d}^{\prime}$, where $\widetilde{\mathfrak{z}}=\operatorname{ker}\left(\left.D^{\prime}\right|_{\mathfrak{z} \cap J_{\mathfrak{z}}}\right)$, $\widetilde{\mathfrak{h}}=\mathbb{R} A \oplus \mathfrak{h}$ and $\mathfrak{d}^{\prime}=\mathfrak{k}^{\prime} \oplus \operatorname{Im}\left(\left.D^{\prime}\right|_{\mathfrak{z} \cap J_{\mathfrak{z}}}\right)$, whenever $D^{\prime} \neq 0$. If $D^{\prime}=0$, we have that $\widetilde{\mathfrak{z}}=\mathbb{R} A \oplus \mathfrak{z}, \widetilde{\mathfrak{h}}=\mathfrak{h}$ and $\mathfrak{d}^{\prime}=\mathfrak{k}^{\prime}$.

7.2. Left-symmetric algebra structures. In this section we show that any unimodular solvable Lie algebra equipped with either a Sasakian or a Vaisman structure admits also another kind of algebraic structure with a geometrical interpretation.

A left-symmetric algebra (LSA) structure on a Lie algebra $\mathfrak{a}$ is a bilinear product $\mathfrak{a} \times \mathfrak{a} \longrightarrow \mathfrak{a},(x, y) \mapsto x \cdot y$, which satisfies

$$
[x, y]=x \cdot y-y \cdot x
$$

and

$$
x \cdot(y \cdot z)-(x \cdot y) \cdot z=y \cdot(x \cdot z)-(y \cdot x) \cdot z,
$$

for any $x, y, z \in \mathfrak{a}$. See [16] for a very interesting review on this subject.

LSA structures have the following well known geometrical interpretation. If $G$ is a Lie group and $\mathfrak{g}$ is its Lie algebra, then LSA structures on $\mathfrak{g}$ are in one-to-one correspondence with left invariant flat torsion-free connections $\nabla$ on $G$. Indeed, this correspondence is given as follows: $\nabla_{x} y=x \cdot y$ for $x, y \in \mathfrak{g}$. Since this connection is left invariant, any quotient $\Gamma \backslash G$ of $G$ by a discrete subgroup $\Gamma$ also inherits a flat torsion-free connection. It is well known that one can study the completeness of the connection $\nabla$ on $G$ in terms only of the corresponding LSA structure on $\mathfrak{g}$. Indeed, $\nabla$ is geodesically complete if and only if the endomorphisms $\mathrm{Id}+\rho(x)$ of $\mathfrak{g}$ are bijective for all $x \in \mathfrak{g}$, where $\rho(x)$ denotes right-multiplication by $x$, i.e., $\rho(x) y=y \cdot x$ (see for instance [49]). In this case, it is said that the LSA structure is complete.

Note that the Levi-Civita connection of a flat metric on a Lie algebra is an example of a complete LSA structure, since the corresponding left invariant metric on any associated Lie group is homogeneous and therefore complete.

Theorem 7.3. Let $(\mathfrak{h},\langle\cdot, \cdot\rangle)$ be a flat Lie algebra and let $\beta$ denote a 2 -form which is parallel with respect to the Levi-Civita connection $\nabla$ of $\langle\cdot, \cdot\rangle$ (hence $\beta$ is closed). Then the central extension $\mathfrak{g}=\mathfrak{h}_{\beta}(\xi)$ admits an LSA structure defined by

$$
(a \xi+x) \cdot(b \xi+y)=\frac{1}{2} \beta(x, y) \xi+\nabla_{x} y, \quad a, b \in \mathbb{R}, x, y \in \mathfrak{h} .
$$

Furthermore, this LSA structure is complete. 
Proof. Taking into account that $\nabla$ is a torsion-free connection on $\mathfrak{h}$ and the fact that $\xi$ is central in $\mathfrak{g}$, it is easily verified that

$$
(a \xi+x) \cdot(b \xi+y)-(b \xi+y) \cdot(a \xi+x)=[a \xi+x, b \xi+y]_{\beta} .
$$

Therefore, (12) holds for this product. In order to prove (13), let us compute

$$
\begin{aligned}
(a \xi+x) \cdot((b \xi+y) \cdot(c \xi+z)) & -(b \xi+y) \cdot((a \xi+x) \cdot(c \xi+z))= \\
& =\frac{1}{2} \beta\left(x, \nabla_{y} z\right) \xi+\nabla_{x} \nabla_{y} z-\frac{1}{2} \beta\left(y, \nabla_{x} z\right) \xi-\nabla_{y} \nabla_{x} z \\
& =-\frac{1}{2} \beta\left(\nabla_{y} x, z\right) \xi+\frac{1}{2} \beta\left(\nabla_{x} y, z\right) \xi+\nabla_{x} \nabla_{y} z-\nabla_{y} \nabla_{x} z \\
& =\frac{1}{2} \beta([x, y], z) \xi+\nabla_{[x, y]} z \\
& =[a \xi+x, b \xi+y]_{\beta} \cdot(c \xi+z),
\end{aligned}
$$

where we have used $\nabla \beta=0$ in the third line and the fact that $\nabla$ is torsion-free and flat in the fourth line. This is equivalent to (13), thus this product is an LSA structure on $\mathfrak{g}$.

Let us prove the completeness. Fix $b \xi+y \in \mathfrak{g}$ and assume that $(\mathrm{Id}+\rho(b \xi+$ $y))(a \xi+x)=0$. Then

$$
\begin{aligned}
0 & =a \xi+x+\frac{1}{2} \beta(x, y) \xi+\nabla_{x} y \\
& =\left(a+\frac{1}{2} \beta(x, y)\right) \xi+\left(x+\nabla_{x} y\right)
\end{aligned}
$$

hence $a+\frac{1}{2} \beta(x, y)=0$ and $x+\nabla_{x} y=0$. But, since $\nabla$ itself is a complete LSA structure on $\mathfrak{h}$, it follows that $x=0$. This implies that $a=0$, and the completeness follows.

COROLlary 7.4. Let $\mathfrak{g}$ be a unimodular solvable Lie algebra.

(1) If $\mathfrak{g}$ carries a Vaisman structure, then $\mathfrak{g}$ admits a complete LSA structure.

(2) If $\mathfrak{g}$ carries a Sasakian structure, then $\mathfrak{g}$ admits a complete LSA structure.

Proof. If $\mathfrak{g}$ admits a Vaisman structure then, according to Theorem 7.1, $\mathfrak{g}$ is a central extension of a coKähler Lie algebra $\mathfrak{d}$ by the fundamental 2 -form $\Phi$, which is parallel. As mentioned in Remark $7.2, \mathfrak{d}$ is flat, and therefore (1) follows from Theorem 7.3.

If $\mathfrak{g}$ admits a Sasakian structure, then it follows from Corollary 3.11 that $\mathfrak{g}$ is a central extension of a Kähler flat Lie algebra. Hence, (2) follows from Theorem 7.3 again.

COROLlary 7.5. Any solvmanifold admitting either an invariant Vaisman structure or an invariant Sasakian structure carries a geodesically complete flat torsion-free connection.

\section{REFERENCES}

[1] D. Alekseevsky, Homogeneous Riemannian spaces of negative curvature, Math. Sbornik, 96 (1975), pp. 93-117. 
[2] D. Alekseevsky, V. Cortés, K. Hasegawa and Y. Kamishima, Homogeneous locally conformally Kähler and Sasaki manifolds, Internat. J. Math., 6 (2015), 1541001 (29 pp).

[3] D. Alekseevsky, K. Hasegawa and Y. Kamishima, Homogeneous Sasaki and Vaisman manifolds of unimodular Lie groups, Nagoya Math. J., (2019), pp. 1-14.

[4] M. A. Álvarez, M. C. Rodríguez-Vallarte and G. Salgado, Contact nilpotent Lie algebras, Proc. Amer. Math. Soc., 145 (2017), pp. 1467-1474.

[5] A. Andrada AND M. ORIGLiA, Locally conformally Kähler structures on unimodular Lie groups, Geom. Dedicata, 179 (2015), pp. 197-216.

[6] A. Andrada, A. Fino And L. Vezzoni, A class of Sasakian 5-manifolds, Transform. Groups, 14 (2009), pp. 493-512.

[7] L. C. de Andrés, L. A. Cordero, M. Fernández and J. J. Mencía, Examples of four dimensional locally conformal Kähler solvmanifolds, Geom. Dedicata, 29 (1989), pp. 227232.

[8] G. Bazzoni, Vaisman nilmanifolds, Bull. Lond. Math. Soc., 49 (2017), pp. 824-830.

[9] M. L. Barberis, I. Dotti AND A. Fino, Hyper-Kähler quotients of solvable Lie groups, J. Geom. Phys., 56 (2006), pp. 691-711.

[10] A. Belgun, On the metric structure of non-Kähler complex surfaces, Math. Ann., 317 (2000), pp. $1-40$.

[11] C. Benson And C. Gordon, Kähler and symplectic structure on nilmanifolds, Topology, 27 (1988), pp. 513-518.

[12] A. Besse, Einstein Manifolds. Classics in Mathematics. Springer, 1987.

[13] D. E. Blair, The theory of quasi-Sasakian structures, J. Differential Geom., 1 (1967), pp. 331345 .

[14] D. E. BLAIR, Riemannian geometry of contact and symplectic manifolds, Progress in Mathematics 203, Birkhäuser, 2002.

[15] C. Bock, On low-dimensional solvmanifolds, Asian J. Math., 20 (2016), pp. 199-262.

[16] D. Burde, Left-symmetric algebras, or pre-Lie algebras in geometry and physics, Cent. Eur. J. Math., 4 (2006), pp. 323-357.

[17] S. Console, G. Ovando And M. Subils, Solvable models for Kodaira surfaces, Mediterr. J. Math., 12 (2015), pp. 187-204.

[18] L. Cordero, M. Fernández And M. DE LÉon, Compact locally conformal Kähler nilmanifolds, Geom. Dedicata, 21 (1986), pp. 187-192.

[19] S. Dragomir and L. Ornea, Locally conformal Kähler geometry. Progress in Mathematics 155, Birkhäuser, 1998.

[20] A. Fino, A. Otal and L. UGarte, Six-dimensional solvmanifolds with holomorphically trivial canonical bundle, Int. Math. Res. Not. IMRN, 24 (2015), pp. 13757-13799.

[21] A. Fino And L. Vezzoni, Some results on cosymplectic manifolds, Geom. Dedicata, 151 (2011), pp. $41-58$.

[22] M. Fischer, Lattices of oscillator groups, J. Lie Theory, 27 (2017), pp. 85-110.

[23] P. Gauduchon, A. Moroianu and L. Ornea, Compact homogeneous lcK manifolds are Vaisman, Math. Ann., 361 (2015), pp. 1043-1048.

[24] A. Gray and L. Hervella, The sixteen classes of almost Hermitian manifolds and their linear invariants, Ann. Mat. Pura Appl., 123 (1980), pp. 35-58.

[25] J. Hano, On Kaehlerian homogeneous spaces of unimodular Lie groups, Amer. J. Math., 79 (1957), pp. 885-900.

[26] K. Hasegawa, Complex and Kähler structures on compact solvmanifolds, J. Symplectic Geom., 3 (2005), pp. 749-767.

[27] K. Hasegawa and Y. Kamishima, Locally conformally Kähler structures on homogeneous spaces. In: Ochiai T., Mabuchi T., Maeda Y., Noguchi J., Weinstein A. (eds) "Geometry and Analysis on Manifolds". Progress in Mathematics, vol 308. Birkhäuser, Cham (2015).

[28] K. Hasegawa and Y. Kamishima, Compact homogeneous locally conformally Kähler manifolds, Osaka J. Math., 53 (2016), pp. 683-703.

[29] T. KASHIWADA AND S. SATO, On harmonic forms in compact locally conformal Kähler manifolds with the parallel Lee form, Ann. Fac. Sci. Univ. Nat. Zaïre (Kinshasa) Sect. Math.Phys., 6 (1980), pp. 17-29.

[30] H. KasuyA, Vaisman metrics on solvmanifolds and Oeljeklaus-Toma manifolds, Bull. Lond. Math. Soc., 45 (2013), pp. 15-26.

[31] H. C. LEE, A kind of even dimensional differential geometry and its application to exterior calculus, Amer. J. Math., 65 (1943), pp. 433-438.

[32] H. Li, Topology of co-symplectic/co-Kähler manifolds, Asian J. Math., 12 (2008), pp. 527-543.

[33] A. Lichnerowicz, Les groupes kählériens, in: Symplectic geometry and mathematical physics (Aix-en-Provence, 1990), pp. 245-259, Progress in Mathematics 99, Birkhäuser, 1991. 
[34] J. C. Marrero And E. PAdrón, Compact generalized Hopf and cosymplectic solvmanifolds and the Heisenberg group H(n,1), Israel J. Math., 101 (1997), pp. 189-204.

[35] A. Medina And P. Revoy, Les groupes oscillateurs et leurs réseaux, Manuscripta Math., 52 (1985), pp. 81-95.

[36] A. Medina, Groupes de Lie munis de métriques bi-invariantes, Tohoku Math. J., 37 (1985), pp. 405-421.

[37] J. Milnor, Curvatures of left invariant metrics on Lie groups, Adv. Math., 21 (1976), pp. 293329.

[38] G. D. Mostow, Factor spaces of solvable groups, Ann. of Math., 60 (1954), pp. 1-27.

[39] K. Oeljeklaus And M. Toma, Non-Kähler compact complex manifolds associated to number fields, Ann. Inst. Fourier (Grenoble), 55 (2005), pp. 161-171.

[40] A. L. OnishchiK And E.B. Vinberg, Lie groups and Lie algebras III, Encyclopaedia of Mathematical Sciences 41, Springer-Verlag Berlin Heidelberg, 1994.

[41] L. OrneA, Locally conformal Kähler manifolds. A selection of results, Lect. Notes Semin. Interdiscip. Mat. IV (2005), pp. 121-152.

[42] L. Ornea And M. Verbitsky, Structure theorem for compact Vaisman manifolds, Math. Res. Lett., 10 (2003), pp. 799-805.

[43] L. Ornea AND M. Verbitsky, LCK rank of locally conformally Kähler manifolds with potential, J. Geom. Phys., 107 (2016), pp. 92-98.

[44] S. Raghunathan, Discrete subgroups of Lie groups, Springer, Berlin, 1972.

[45] H. SAWAI, Locally conformal Kähler structures on compact nilmanifold with left-invariant complex structures, Geom. Dedicata, 125 (2007), pp. 93-101.

[46] H. SAWAI, Locally conformal Kähler structures on compact solvmanifolds, Osaka J. Math., 49 (2012), pp. 1087-1102.

[47] H. SAWAI, Vaisman structures on compact solvmanifolds, Geom. Dedicata, 178 (2015), pp. 389404.

[48] H. SAWAI, Structure theorem for Vaisman completely solvable solvmanifolds, J. Geom. Phys., 114 (2017), pp. 581-586.

[49] D. Segal, The structure of complete left-symmetric algebras, Math. Ann., 293 (1992), pp. 569578.

[50] I. VAISMAn, Locally conformal Kähler manifolds with parallel Lee form, Rend. Mat., VI Ser., 12 (1979), pp. 263-284.

[51] I. Vaisman, Generalized Hopf manifolds, Geom. Dedicata, 13 (1982), pp. 231-255.

[52] I. VAISMAN, On locally conformal almost Kähler manifolds, Israel J. Math., 24 (1976), pp. 3-4.

[53] M. Verbitsky, Theorems on the vanishing of cohomology for locally conformally hyper-Kähler manifolds, Proc. Steklov Inst. Math., 246 (2004), pp. 54-78. 
A. ANDRADA AND M. ORIGLIA 